\title{
Strongly Coupled Computation of Material Response and Nonequilibrium Flow for Hypersonic Ablation
}

\author{
Alexandre Martin* \\ University of Kentucky, Lexington, Kentucky 40506 \\ and \\ Iain D. BoydI \\ University of Michigan, Ann Arbor, Michigan 48109 \\ DOI: $\underline{10.2514 / 1 . A 32847}$
}

\begin{abstract}
The steps necessary to achieve the strong coupling between a flowfield solver and a material response solver are presented. This type of coupling is required to accurately capture the complex aerothermodynamic physics occurring during hypersonic atmospheric entries. A blowing boundary condition for the flowfield solver is proposed. This allows the ablating gas calculated by the material response solver to be correctly injected in the boundary layer. A moving mesh algorithm for the flowfield solver that implicitly enforces the geometric conservation law is presented. Using that capability, a mesh movement procedure for surface recession and for accurate shock capturing is proposed. The entire technique is tested using a material response solver with surface ablation and pyrolysis coupled to a hypersonic solver for weakly ionized flows in thermochemical nonequilibrium. Results using the reentry trajectory of the IRV-2 test vehicle are presented, showing that the surface heat fluxes remain accurate as the vehicle geometry and freestream conditions change.
\end{abstract}

\begin{tabular}{|c|c|c|}
\hline & & Nomenclature \\
\hline $\boldsymbol{A}$ & $=$ & Jacobian matrix \\
\hline$a$ & $=$ & speed of sound, $\mathrm{m} / \mathrm{s}$ \\
\hline$C$ & $=$ & source term vector \\
\hline E & $=$ & total energy, $\mathrm{J} / \mathrm{kg}$ \\
\hline $\boldsymbol{e}$ & $=$ & internal energy vector, $\mathrm{J} / \mathrm{kg}$ \\
\hline $\boldsymbol{F}$ & $=$ & inviscid flux tensor \\
\hline $\boldsymbol{F}_{d}$ & $=$ & diffusive flux tensor \\
\hline Fo & $=$ & Forchheimer number \\
\hline$G$ & $=$ & flux tensor, $\boldsymbol{F}-\boldsymbol{F}_{d}-\boldsymbol{u} \boldsymbol{U}$ \\
\hline$h$ & $=$ & enthalpy, J/kg \\
\hline $\boldsymbol{h}$ & $=$ & species enthalpy vector, $\mathrm{J} / \mathrm{kg}$ \\
\hline$h_{r}$ & $=$ & recovery enthalpy, $\mathrm{J} / \mathrm{kg}$ \\
\hline$I^{\prime}$ & $=$ & identity matrix \\
\hline$J$ & $=$ & directional species diffusion flux tensor, $\mathrm{kg} / \mathrm{m}^{3}$ \\
\hline$K$ & $=$ & permeability, $\mathrm{m}^{2}$ \\
\hline$L$ & $=$ & eigenvector similarity transformation matrix \\
\hline$\ell^{2}$ & $=$ & euclidian norm \\
\hline$l$ & $=$ & characteristic length, $\mathrm{m}$ \\
\hline$\dot{m}^{\prime \prime \prime}$ & $=$ & volumetric mass source term, $\mathrm{kg} / \mathrm{m}^{3} \cdot \mathrm{s}$ \\
\hline$\dot{m}^{\prime \prime}$ & $=$ & mass rate $\mathrm{kg} / \mathrm{m}^{2} \cdot \mathrm{s}$ \\
\hline$p$ & $=$ & pressure, $\mathrm{Pa}$ \\
\hline$p_{\eta}$ & $=$ & total pressure, $\mathrm{Pa}$ \\
\hline$q$ & $=$ & surface heat transfer rate, $\mathrm{W} / \mathrm{m}^{2}$ \\
\hline$\dot{q}^{\prime \prime}$ & $=$ & internal heat flux, $\mathrm{W} / \mathrm{m}^{2}$ \\
\hline $\boldsymbol{R}$ & $=$ & right-hand side term \\
\hline $\boldsymbol{r}$ & $=$ & distance vector, $\mathrm{m}$ \\
\hline$S, S$ & $=$ & surface, $\mathrm{m}^{2}$ \\
\hline$T$ & $=$ & temperature, $\mathrm{K}$ \\
\hline
\end{tabular}

Part of this work was Presented as Paper 2010-5062 at the 10th AIAA/ ASME Joint Thermophysics and Heat Transfer Conference, Chicago, IL, 28 June-1 July 2010; received 26 September 2013; revision received 29 May 2014; accepted for publication 27 July 2014; published online 29 October 2014. Copyright $\odot 2014$ by Alexandre Martin and Iain D. Boyd. Published by the American Institute of Aeronautics and Astronautics, Inc., with permission. Copies of this paper may be made for personal or internal use, on condition that the copier pay the $\$ 10.00$ per-copy fee to the Copyright Clearance Center, Inc., 222 Rosewood Drive, Danvers, MA 01923; include the code 1533-6794/ 14 and $\$ 10.00$ in correspondence with the CCC.

*Assistant Professor, Department of Mechanical Engineering, 261 Ralph G. Anderson Building; also Associate Faculty, Center for Computational Science. Associate Fellow AIAA.

$\dagger$ James E. Knott Professor of Engineering, Department of Aerospace Engineering, 3000 Francois-Xavier Bagnoud Building. Fellow AIAA.

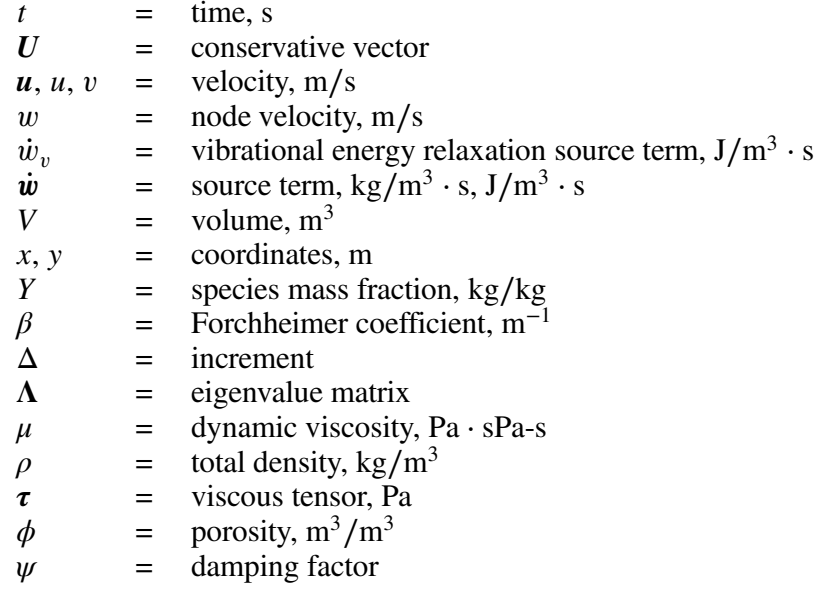

Subscripts

$\begin{array}{lll}c & =\text { char } \\ \mathrm{cs} & =\text { control surface } \\ \mathrm{cv} & =\text { control volume } \\ e & =\text { electron-electronic } \\ f & =\text { fixed mesh } \\ g & =\text { gas } \\ n & =\text { normal } \\ r & =\text { rotational } \\ r & =\text { recovery } \\ s & =\text { solid, volume increment } \\ \boldsymbol{T} & =\text { transposed } \\ v & =\text { virgin, vibrational } \\ w & =\text { wall } \\ x, y, z & =\text { coordinates } \\ 0 & =\text { nonblowing } \\ \infty & =\text { freestream }\end{array}$

\section{Introduction}

W HETHER it is for planetary exploration or for atmospheric cruise, all spacecraft traveling at high velocity require a thermal protection system (TPS). Materials used for TPSs are diverse and depend on a multitude of parameters such as flight path, atmospheric conditions, and peak velocity. In general, they can be classified in two 
broad categories: reusable material, such as the tiles of the shuttle, or nonreusable material, such as the one used on the Mars Science Laboratory entry craft. The theory behind the use of nonreusable materials is based on the assumption that the energy used to remove layers of materials at the surface of the vehicle does not penetrate further into the material and therefore attenuates the temperature increase of the vehicle.

Modeling of nonreusable material effectively started at the beginning of the space program and later came to a halt with the rise of the shuttle program. The space exploration objectives elaborated in the last 20 years have, however, resulted in an increased interest in the subject. With the fast advance of computer speed as well as numerical algorithms, it is now possible to significantly increase the accuracy and fidelity of atmospheric reentry ablation modeling. In general, it involves accounting for the physics present in three distinct regions: 1) the external flowfield, 2) the inside of the TPS material, and 3) the surface between the the two. Figure 1 illustrates these three regions, also listing the phenomena of interest in each of them.

Historically, TPSs have been modeled using stand-alone material response (MR) codes that use inputs from trajectory databases populated using computational fluid dynamics (CFD) solutions [1]. Boundary-layer approximation and heat transfer coefficient models were used as uncoupled boundary conditions in the MR solver [2]. This approach proved to be efficient, but not necessarily accurate, considering the large number of assumptions present in the surface balance models. To increase the accuracy and remove the uncertainties linked to these assumptions, it is therefore necessary to properly model the heat rate at the surface of the vehicle. To do so, the ablating boundary condition must take into account many phenomena: surface recession, wall temperature, blowing rates, gas composition, surface chemistry, etc. One way to account for effects of the TPS on the surface flow is to link a material response model to the flow solver.

To do this, modifications to the flow solver are first needed, especially at the wall, where ablating gases need to be introduced in the flowfield. Moreover, to dynamically account for the effects of the surface recession on the flowfield, the mesh must be allowed to move as the surface ablates. The present study describes in detail the steps that are required to modify a hypersonic flow solver so that it can be strongly coupled to a material response code.

To demonstrate the procedure, the material response code MOPAR [3] is coupled to the hypersonic aerothermodynamics code LeMANS [4]. Verifications are presented for each individual modification, showing excellent agreement to analytical solutions, empirical relations, and other published data. To demonstrate the capability of the new coupled model, a simulation of a generic reentry of the IRV-2 vehicle is presented. This test case highlights the necessity to perform a mesh adaptation that improves the solution by aligning the cells of the mesh to the shock.

\section{LeMANS: Unstructured Tridimensional Navier- Stokes Solver for Hypersonic Nonequilibrium Aerothermodynamics}

\section{A. Overview}

The CFD code used in the present analysis is LeMANS, a fully implicit finite-volume aerothermodynamic Navier-Stokes solver [4]. The code accounts for chemical nonequilibrium, using finite-rate chemistry, and thermal nonequilibrium. The various energy modes can be coupled together, allowing to up to four different temperatures (translational $T$, vibrational-electronic $T_{v e}$, rotational $T_{r}$, and electron $T_{e}$ ) [5]. The viscous tensor is obtained assuming a Newtonian fluid and Stokes hypothesis, and the diffusion fluxes are modeled using Fick's law. The transport properties are computed using the semi-empirical Wilke mixing rule, with individual species viscosity obtained by curve fits, and the conductivity using Eucken's relation. An equal diffusion coefficient is used for all species, calculated assuming a constant Lewis number. Fourier's law is used to compute the conductive heat fluxes for each individual temperature.

The code is fully implicit, using the data-parallel line relaxation method for accelerated convergence. The code is also massively parallel and can therefore take advantage of parallel computing using domain decomposition. To compute the inviscid flux, a modified version of the Steger-Warming flux vector splitting scheme is used; this scheme has been chosen because of its low dissipation and its accuracy in the boundary layer. The code has been extensively verified and validated, both using experimental data as well as codeto-code comparison, using published results from NASA Ames Research Center's DPLR [6], NASA Langley Research Center's LAURA [7], and University of Minnesota's US3D [8] .

\section{B. Governing Equations}

With the approximations mentioned previously, the conservation equations for a three-dimensional system are written as

$$
\frac{\partial \boldsymbol{U}}{\partial t}+\nabla \cdot\left(\boldsymbol{F}-\boldsymbol{F}_{d}\right)=\boldsymbol{C}
$$

where

$$
\boldsymbol{U}=\left(\begin{array}{c}
\rho \boldsymbol{Y}^{T} \\
\rho \boldsymbol{u}^{T} \\
E \\
E_{v e} \\
E_{r}
\end{array}\right) \quad \text { and } \quad \boldsymbol{C}=\left(\begin{array}{c}
\dot{\boldsymbol{w}}^{T} \\
\mathbf{0}^{T} \\
0 \\
\dot{w}_{v} \\
\dot{w}_{r}
\end{array}\right)
$$

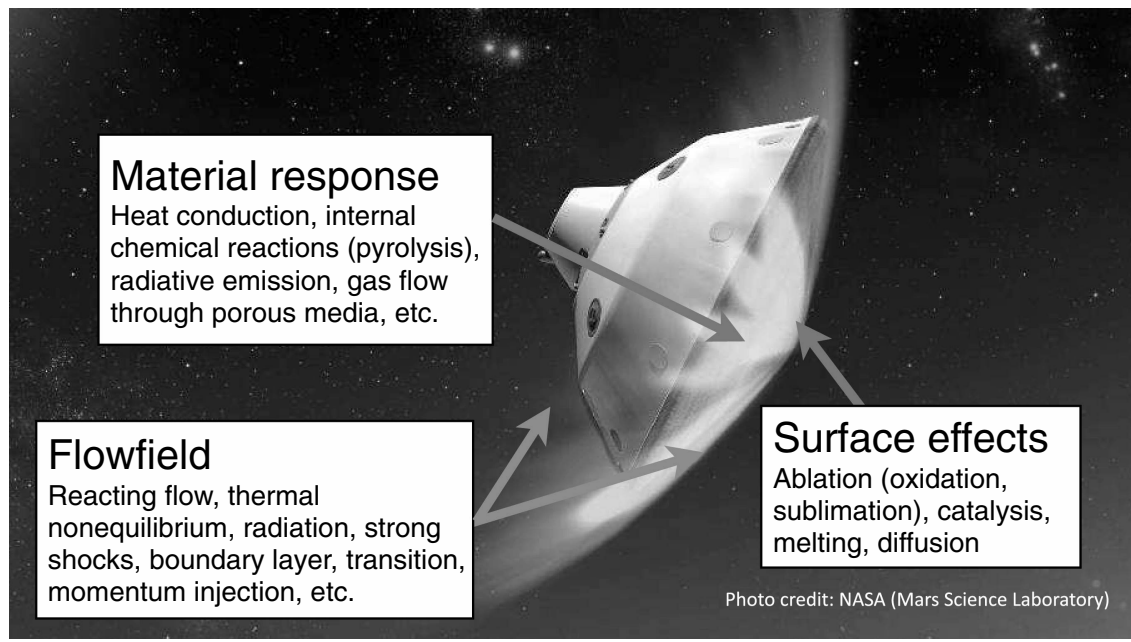

Fig. 1 Some of the main physical phenomena occurring in an atmospheric entry. 
are the vector of conserved variables and the vector of source terms, respectively. In these expressions, $\boldsymbol{Y}=\left(Y_{1}, \ldots, Y_{n s}\right)$ is the species densities vector; $\boldsymbol{u}$ is the bulk velocity components; $E, E_{v e}$, and $E_{r}$ are the total, the vibrational-electron-electronic, and the rotational energy per unit volume of mixture, respectively.

The inviscid and diffusive flux matrices are given by

$$
\boldsymbol{F}=\left(\begin{array}{c}
\rho \boldsymbol{Y}^{T} \boldsymbol{u} \\
\rho \boldsymbol{u}^{T} \boldsymbol{u}+\boldsymbol{I} p \\
(E+p) \boldsymbol{u} \\
E_{v e} \boldsymbol{u} \\
E_{r} \boldsymbol{u}
\end{array}\right) \text { and } \boldsymbol{F}_{d}=\left(\begin{array}{c}
-\boldsymbol{J} \\
\boldsymbol{\tau} \\
\boldsymbol{u} \boldsymbol{\tau}-\left(\boldsymbol{q}_{t}+\boldsymbol{q}_{r}+\boldsymbol{q}_{v e}\right)-\left(\boldsymbol{h}^{T} \boldsymbol{J}\right) \\
-\boldsymbol{q}_{v e}-\left(\boldsymbol{e}_{v e}^{T} \boldsymbol{J}\right) \\
-\boldsymbol{q}_{r}-\left(\boldsymbol{e}_{r}^{T} \boldsymbol{J}\right)
\end{array}\right)
$$

where $p$ is the pressure; $\boldsymbol{\tau}$ is the viscous tensor; and $\boldsymbol{q}_{t}, \boldsymbol{q}_{r}$, and $\boldsymbol{q}_{v e}$ are the directional translational, rotational, and vibrational-electronelectronic heat fluxes vectors, respectively. Moreover, $\boldsymbol{h}$ is the species enthalpy vector, and $\boldsymbol{J}$ is the directional species diffusion flux tensor. More details on these equations and on the modeling of the individual terms can be found in [9].

Equation (1) can be reduced to a simple vector form that splits the flux tensor using the conservative vector,

$$
\begin{gathered}
\frac{\partial}{\partial t}\left(\begin{array}{c}
\rho \boldsymbol{Y}^{T} \\
\rho \boldsymbol{u}^{T} \\
E \\
E_{v e} \\
E_{r}
\end{array}\right)+\nabla \cdot\left[\left(\begin{array}{c}
\rho \boldsymbol{Y}^{T} \\
\rho \boldsymbol{u}^{T} \\
E \\
E_{v e} \\
E_{r}
\end{array}\right) \boldsymbol{u}+\left(\begin{array}{c}
\mathbf{0} \\
\boldsymbol{I} p \\
\boldsymbol{u} p \\
\mathbf{0} \\
\mathbf{0}
\end{array}\right)\right. \\
-\left(\begin{array}{c}
\boldsymbol{u} \boldsymbol{\tau}-\left(\boldsymbol{q}_{t}+\boldsymbol{q}_{r}+\boldsymbol{q}_{v e}\right)-\left(\boldsymbol{h}^{T} \boldsymbol{J}\right) \\
-\boldsymbol{q}_{v e}-\left(\boldsymbol{e}_{v e}^{T} \boldsymbol{J}\right) \\
-\boldsymbol{q}_{r}-\left(\boldsymbol{e}_{r}^{T} \boldsymbol{J}\right)
\end{array}\right)=\left(\begin{array}{c}
\dot{\boldsymbol{w}}^{T} \\
\mathbf{0}^{T} \\
0 \\
\dot{w}_{v} \\
\dot{w}_{r}
\end{array}\right)
\end{gathered}
$$

which can be rereduced to

$$
\frac{\partial \boldsymbol{U}}{\partial t}+\nabla \cdot(\boldsymbol{U} \boldsymbol{u}+\boldsymbol{G})=\boldsymbol{C}
$$

\section{MOPAR: Material Response Code}

The material response code used in this validation is called MOPAR. The code employs the same methodology as the ones described in Blackwell and Hogan [10], Amar et al. [11] and Amar et al. [12] uses the control volume finite-element method to model surface ablation with wall recession, as well as inner decomposition and pyrolysis gas behavior. The model is described by the following four governing equations:

1) The mixture energy equation is

$$
\frac{\mathrm{d}}{\mathrm{d} t} \int_{\mathrm{cv}} \rho E \mathrm{~d} V-\int_{\mathrm{cs}} \rho h v_{\mathrm{cs}} \mathrm{d} S+\int_{\mathrm{cs}} \phi \rho_{g} h_{g} v_{g} \mathrm{~d} S+\int_{\mathrm{cs}} \dot{q}^{\prime \prime} \mathrm{d} A=0
$$

2) The solid phase equation is

$$
\frac{\mathrm{d}}{\mathrm{d} t} \int_{\mathrm{cv}} \rho_{s} \mathrm{~d} V-\int_{\mathrm{cs}} \rho_{s} v_{\mathrm{cs}} \mathrm{d} S-\int_{\mathrm{cv}} \dot{m}_{s}^{\prime \prime \prime} \mathrm{d} V=0
$$

3) The gas-phase continuity equation is

$$
\frac{\mathrm{d}}{\mathrm{d} t} \int_{\mathrm{cv}} \phi \rho_{g} \mathrm{~d} V-\int_{\mathrm{cs}} \phi \rho_{g} v_{\mathrm{cs}} \mathrm{d} S+\int_{\mathrm{cs}} \phi \rho_{g} v_{g} \mathrm{~d} S-\int_{\mathrm{cv}} \dot{m}_{g}^{\prime \prime \prime} \mathrm{d} V=0
$$

4) The momentum equation, Forchheimer's law is

$$
\frac{\partial P}{\partial x}=-\frac{\mu}{K} \phi v_{g}(1+F o)
$$

The first terms in Eqs. (ㅁ-8) account for the energy, solid mass, and gas mass content, respectively, and the second terms account for the grid convection. The third terms in Eqs. (6) and (ㅁ) are the gas fluxes, and the last terms in Eqs. (7) to (8) are the source terms. As for the last term of Eq. (6), it accounts for the heat conduction within the solid. In Eq. (9), Forchheimer's law, the Forchheimer number Fo is given by

$$
F o=\frac{\beta K \rho \phi v_{g}}{\mu}
$$

This number indicates when microscopic effects (pore size) are perceivable at a macroscopic (geometry size) level. In this formulation, it is easy to see that when $F o \ll 1$ the equation simply reduces to Darcy's law. Therefore, it is more logical to use the Forchheimer number to predict non-Darcian flow and is thus more rigorous to use Forchheimer's law.

Equations (6) and () are solved implicitly using Landau coordinates, which allows the grid to contract or expand. The equations are solved sequentially using Newton's method for nonlinear systems. Equation (7) is solved analytically for $\rho_{s}$ and integrated into the previous two equations. As for Eq. (9), Forchheimer's law [13], it is used to directly obtain $v_{g}$ and then used in the gas-phase continuity equation.

The code also takes into account multiple coordinate systems (cylindrical and spherical) and could be used to solve a problem involving ablation on both sides of the system, using a new tridiagonal solver [14]. The code has been extensively validated over the years, using experimental data [3] and code-to-code validations $[15,16]$.

\section{Modifications to Flow Solver}

\section{A. Blowing Boundary Conditions}

Because of the coupling, momentum injection from the material response is introduced in the flowfield, at the surface of the vehicle. To accommodate this physical phenomenon, a modification to the surface boundary condition of the CFD code is necessary. To implement this blowing boundary, the diffusive and convective fluxes are computed at the surface. The resulting equation, called the surface momentum balance $(\mathrm{SMoB})$, is therefore obtained by equating the fluxes normal to the surface from the gas phase (subscript $n c$ ) to the fluxes from the material phase (subscript $w$ ). By neglecting the diffusive fluxes [17], the following expression is obtained:

$$
p_{\eta}=p_{n c}+\rho_{n c} v_{n c}^{2}=p_{w}\left(\rho_{w}, T_{w}\right)+\rho_{w} v_{w}^{2}
$$

The $\mathrm{SMoB}$ is generally neglected in CFD simulations using ablating boundary conditions [18], or simply reduced to an equal pressure relation. The physical values that are imposed at the wall are the temperature $T_{w}$, the blowing mass flow rate $\dot{m}_{w}^{\prime \prime}=\rho_{w} v_{w}$, and the species mass fractions $Y_{w i}$. It is to be noted that these values will eventually be obtained by solving the surface mass balance (SMB) equation for $Y_{w i}$ and $\dot{m}_{w}^{\prime \prime}$ and the surface energy balance equation (SEB) for $T_{w}$ [18]. In the current approach, the SMB and SEB are solved in the material response code, and the values of $T_{w}, Y_{w i}$, and $\dot{m}_{w}^{\prime \prime}$ are transferred to the CFD code by way of the coupling scheme described in Sec. VI. 
Using the perfect gas relation at the wall, the equations can be rearranged to obtain the primitive variables:

$$
\begin{aligned}
& \rho_{w}=\frac{p_{\eta}+\sqrt{\left.p_{\eta}^{2}-4 R T_{w} \dot{m}_{w}^{\prime \prime 2}\right)}}{2 R T_{w}} \\
& v_{w}=\frac{2 R T_{w} \dot{m}_{w}^{\prime \prime}}{p_{\eta}+\sqrt{\left.p_{\eta}^{2}-4 R T_{w} \dot{m}_{w}^{\prime \prime 2}\right)}} \\
& p_{w}=\frac{p_{\eta}+\sqrt{\left.p_{\eta}^{2}-4 R T_{w} \dot{m}_{w}^{\prime \prime 2}\right)}}{2}
\end{aligned}
$$

Once these values are computed, the conservative quantities in the ghost cells of the boundary are set such that the flux across the wall is the required blowing flux. This blowing boundary condition has been tested over a wide range of blowing rates, assuring the robustness of the implementation. Following the methodology for the verification and validations of NASA Ames Research Center's DPLR code [19] and NASA Langley Research Center's LAURA code [17], the blowing boundary of LeMANS is also verified and validated.

\section{Blasius Analytical Solution}

As a first verification, a simulation of the Blasius analytical approximation for an airflow over a flat plate with blowing and suction is performed. In this approximation, the analytical solution is obtained by solving

$$
f^{\prime \prime \prime}+f f^{\prime \prime}=0
$$

where $f^{\prime}(\eta)=2 \frac{u}{u_{\infty}}$, with $\eta=\frac{1}{2} \sqrt{\operatorname{Re}_{x}} \frac{y}{x}$ and $R e_{x}=\frac{u_{\infty} x}{\nu}$. The mass flow rate is imposed directly in LeMANS, using the following relation:

$$
\dot{m}_{w}^{\prime \prime}=\rho_{\infty} u_{\infty} \frac{-f(0)}{2 \sqrt{R e_{x}}}
$$

To insure that no perturbation arises from the inlet and outlet boundary conditions and that the flow is allowed to fully develop after and before the $1 \mathrm{~m}$ flat plate, a large computation domain of $10 \mathrm{~m}$ in all directions is used. The freestream conditions are set to normal pressure and temperature, at a velocity of Mach 0.3 , which is the limit of the incompressible flow assumption and therefore the limit of the Blasius solution. Figure 2 shows the comparison between the simulation and the analytical solution tabulated by [20] for the regular Blasius profile $(f(0)=0)$, blowing $(f(0)=-0.4,-0.8)$, and suction $(f(0)=1.2,5.0)$. Even though there are minor discrepancies between both solutions, either caused by the incompressibility assumptions of the Blasius solution or the numerical dissipation induced by the Steger-Warming scheme, the general trends of the curves show good agreement and are sufficiently close to confirm the correct implementation.

\section{Kays and Crawford's Blowing Correction on Heat Transfer}

To take into account the heat flux reduction caused by the blowing in the boundary layer, Kays and Crawford [21] established a simple relation,

$$
\Omega_{b w}=\frac{q}{q_{0}}=\frac{\ln \left(B^{\prime}+1\right)}{B^{\prime}}
$$

which was later extended to include the fitting parameter $\lambda$,

$$
\Omega_{b w}=\frac{\ln \left(2 \lambda B^{\prime}+1\right)}{2 \lambda B^{\prime}}
$$

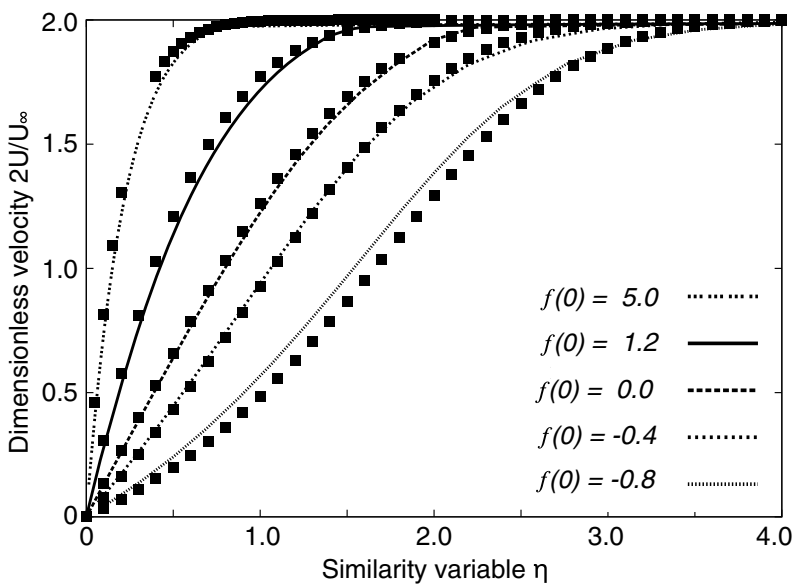

Fig. 2 Comparison between analytical (symbols) and numerical (lines) results for the Blasius blowing boundary conditions.

where

$$
B^{\prime}=\frac{\dot{m}_{w}^{\prime \prime}}{\rho_{e} u_{e} S t}
$$

is the nondimensionalized blowing rate, calculated using the Stanton number,

$$
S t=\frac{q}{\rho_{e} u_{e}\left(h_{r}-h_{w}\right)}
$$

The parameter $\lambda$ usually takes the value of 0.4 for laminar flows and 0.5 for turbulent flows; however, it has recently been suggested that these values might not be accurate across different velocity regimes [19]. Nevertheless, the equation can be used to validate the general trends of the heat flux. To do so, four different Couette flow test cases are run; the detailed conditions of each simulation are presented in Table 1 . As can be seen in Figs. 3 and $\underline{4}$, the simulation results obtained with LeMANS agree with the empirical relation for all cases. However, it is to be noted that none of the simulations have a close fit to the $\lambda=0.4$ analytical solution; 0.5 and 0.6 are a better match for subsonic flows, and 0.3 is a better match for supersonic flows. It is also interesting to note the difference in the general trend of the curves for the subsonic and supersonic regimes.

\section{B. Moving Mesh}

Ablation causes the surface of the vehicle to recess, which means that the computational mesh needs to either be regenerated at every trajectory point or allowed to move as the computation is being made. To be able to achieve a stronger coupling scheme, the latter method is needed. The method chosen, initially proposed by [22], solves implicitly the geometric conservation law (GCL) in the discretized governing equations. The GCL states that the volumetric increase of a control volume during an interval $\Delta t$ must be equal to the total volume swept by the movement of the boundaries of the control volume during that period. This translates to the following equation:

$$
\frac{V^{n+1}-V^{n}}{\Delta t}=\sum_{k} \frac{\delta V_{k}}{\Delta t}
$$

Table 1 Test conditions for the heat transfer blowing correction verification (air)

\begin{tabular}{lccccc}
\hline \hline Test case & $u_{\infty}, \mathrm{m} / \mathrm{s}$ & $T_{\infty}, \mathrm{K}$ & $\rho_{\infty}, \mathrm{kg} / \mathrm{m}^{3}$ & $T_{w}, \mathrm{~K}$ & $\dot{m}_{w}^{\prime \prime}, \mathrm{kg} / \mathrm{m}^{2}-\mathrm{s}$ \\
\hline 1 & 0.102 & 1490 & 1.18 & 298 & 0.005 \\
2 & 0.102 & 1490 & 1.18 & 298 & 0.05 \\
3 & 1.02 & 1490 & 0.118 & 298 & 0.005 \\
4 & 1.02 & 1490 & 0.118 & 298 & 0.05 \\
\hline \hline
\end{tabular}




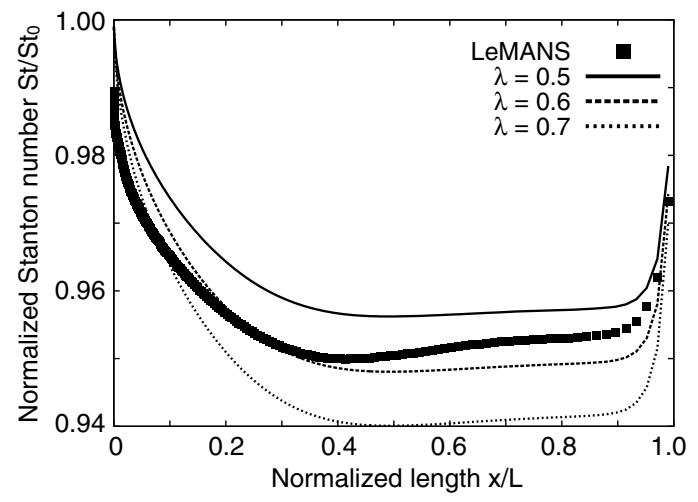

a) Test case 1

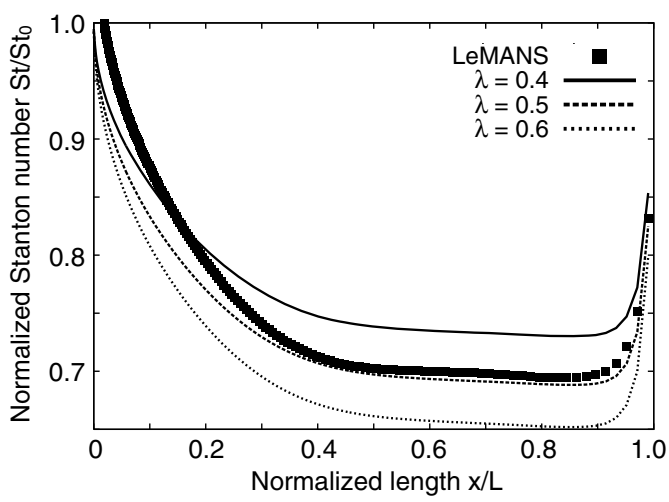

b) Test case 2

Fig. 3 Subsonic (Mach 0.13) laminar blowing correction for test cases 1 and 2 of Table $\underline{1}$.

The chosen method uses an implicit formulation in which the GCL is directly integrated in the governing equations, and not solved on its own. This has the advantage of being valid for both explicit and implicit schemes, works on any kind of mesh cells, is easy to implement in a finite-volume scheme developed for fixed meshes, and retains the order of accuracy of the scheme.

To implement the method, the flow solver must be modified when performing the computation of the fluxes, as well as during time integration.

\section{Implicit Geometric Constriction Law Formulation}

Integrated over an arbitrary volume $V$, Eq. (5) can be written for an arbitrarily moving system, which is known as the arbitrary Lagrangian-Eulerian (ALE) formulation,

$$
\frac{\partial}{\partial t} \int_{\mathrm{cv}} \boldsymbol{U} \mathrm{d} V+\oint_{\mathrm{cs}}(\boldsymbol{U} \boldsymbol{u}-\boldsymbol{U} \boldsymbol{w}+\boldsymbol{G}) \cdot \mathrm{d} \boldsymbol{S}=\int_{\mathrm{cv}} \boldsymbol{C} \mathrm{d} V
$$

where $S$ is the vector area of surface cs, which is one of the enclosing surfaces of the time-dependent volume cv; $\boldsymbol{u}$ is the velocity of the fluid; and $\boldsymbol{w}$ is the given velocity of cs.

According to [22], the discretization of the governing equation for a finite-volume scheme is

$$
V^{n+1} \boldsymbol{U}^{n+1}-V^{n} \boldsymbol{U}^{n}=-\sum_{k} \boldsymbol{F}_{n, k} S_{k} \Delta t+V \boldsymbol{C} \Delta t
$$

where $\boldsymbol{F}_{n}=\boldsymbol{F} \boldsymbol{n}^{T}$, of which $\boldsymbol{n}=\left(\begin{array}{lll}n_{x} & n_{y} & n_{z}\end{array}\right)$ is the normal vector of the face $S$. When applied to Eq. ()ㅡ, the following is obtained

$$
\boldsymbol{F}_{n}=\boldsymbol{U}\left(u_{n}-\bar{w}_{n}\right)+\boldsymbol{G} \boldsymbol{n}^{T}
$$

where

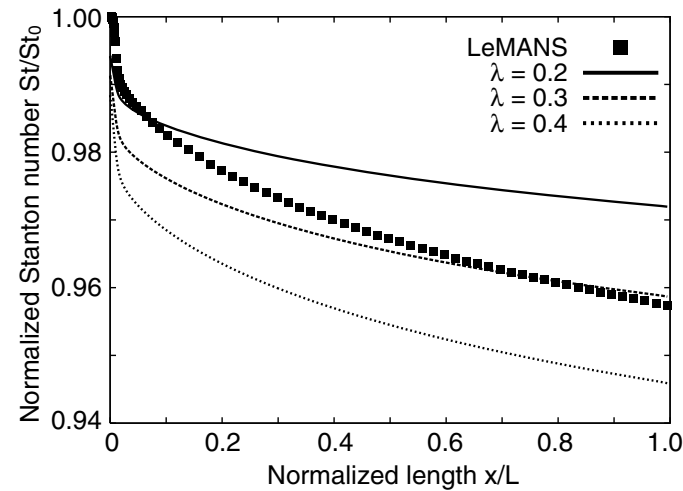

a) Test case 3

$$
\bar{w}_{n}=\frac{\Delta V}{S \Delta t}
$$

and where $\Delta V$ is the face volumetric increment calculated according to the type of elements. These quantities are calculated in such a way that the volumetric increment is balanced by the flux generated by the face movement, so that in the end the conserved quantity remains unchanged by the moving mesh. For a planar two-dimensional geometry, the volume increment is

$$
\Delta V=\Delta t \boldsymbol{w}_{0} \times \Delta \boldsymbol{r}_{12}^{n+1 / 2}
$$

where $\boldsymbol{w}_{0}$ is the average velocity of the two nodes of the side and $\boldsymbol{r}_{12}^{n+1 / 2}$ is the time-averaged side vector. For an axisymmetric geometry, the volume becomes

$$
\begin{gathered}
\Delta V=\frac{\Delta t}{6}\left(2 y_{1}^{n+1 / 2}+y_{2}^{n+1 / 2}\right) \boldsymbol{w}_{1} \times \Delta \boldsymbol{r}_{12}^{n} \\
+\frac{\Delta t}{6}\left(2 y_{2}^{n+1 / 2}+y_{1}^{n+1 / 2}\right) \boldsymbol{w}_{2} \times \Delta \boldsymbol{r}_{12}^{n} \\
+\frac{\Delta t^{2}}{4}\left(y_{1}^{n+2 / 3}+y_{2}^{n+2 / 3}\right) \boldsymbol{w}_{1} \times \boldsymbol{w}_{2}
\end{gathered}
$$

For three-dimensional tetrahedron volumes, the expression is

$$
\Delta V=\Delta t \boldsymbol{w}_{0} \cdot \boldsymbol{S}^{n+1 / 2}+\frac{\Delta t^{3}}{24} \boldsymbol{w}_{1} \cdot \boldsymbol{w}_{2} \times \boldsymbol{w}_{3}
$$

where $\boldsymbol{w}_{0}$ is now the average velocity of the three nodes of the side and $\boldsymbol{S}=\frac{1}{2} \Delta \boldsymbol{r}_{12} \times \Delta \boldsymbol{r}_{13}$. This expression takes into account the fact that in three dimensions the order in which the nodes are moved leads to different facial volumes. This expression is therefore an average of all the possible movement combinations. It is to be noted that this last

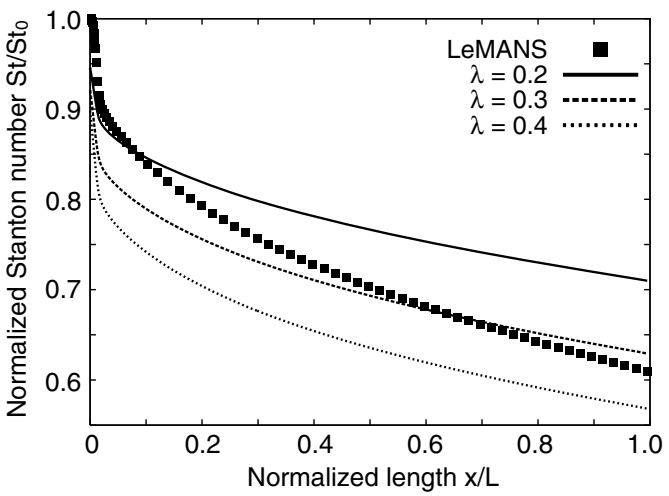

b) Test case 4

Fig. 4 Supersonic (Mach 1.3) laminar blowing correction for test cases 3 and 4 of Table 1. 
equation can be used for any three-dimensional volumetric cells by dividing them into multiple tetrahedrons.

\section{Flux Splitting}

The Jacobian matrix needed to compute the Steger-Warming flux splitting scheme used by the inviscid part of the governing equations is

$$
\boldsymbol{A}=\boldsymbol{A}^{f}-\boldsymbol{I} \bar{w}_{n}
$$

where superscript $f$ refers to the value calculated for a fixed mesh. The eigenvalue matrix of $\boldsymbol{A}$ is therefore

$$
\Lambda=\Lambda^{f}-\boldsymbol{I} \bar{w}_{n}
$$

As for the similarity transformation matrix $\boldsymbol{L}$, constructed from the eigenvectors, it is identical to the one calculated for a fixed mesh:

$$
\boldsymbol{L}=\boldsymbol{L}^{f}
$$

This development shows that, in order to add a moving grid capability to the flux splitting scheme of LeMANS, only the eigenvalues need to be modified.

\section{Implicit Time Integration}

For the types of hypersonic problems solved using LeMANS, an implicit time integration is necessary to take advantage of the larger allowable time steps. Using a Taylor expansion on Eq. (22), the implicit time integration becomes

$$
\boldsymbol{R}^{t+1}=\boldsymbol{R}^{t}+\left[\frac{\partial \boldsymbol{R}}{\partial \boldsymbol{U}}\right]^{t}\left(\boldsymbol{U}^{t+1}-\boldsymbol{U}^{t}\right)+\left[\frac{\partial \boldsymbol{R}}{\partial V}\right]^{t}\left(V^{t+1}-V^{t}\right)
$$

where $-\boldsymbol{R}$ is the right-hand side of Eq. (22). After some manipulation, the time integration becomes

$$
\begin{aligned}
\boldsymbol{U}^{t+1} & =\boldsymbol{U}^{t} \\
+ & {\left[\frac{V^{t}+\Delta V}{\Delta t} \boldsymbol{I}+\left[\frac{\partial \boldsymbol{R}}{\partial \boldsymbol{U}}\right]^{t}\right]^{-1}\left(-\boldsymbol{R}^{t}-\left[\frac{\partial \boldsymbol{R}}{\partial V}\right]^{t} \Delta V-\boldsymbol{U}^{t} \frac{\Delta V}{\Delta t}\right) }
\end{aligned}
$$

It is to be noted that, in order to balance the flux, it is necessary to evaluate the source term using the volumetric time increment:

$$
\boldsymbol{R}^{t}=\sum_{k} \boldsymbol{F}_{n, k}^{t} S_{k}^{t}-\left(V^{t}+\Delta V\right) \boldsymbol{C}
$$

\section{Courant-Friedrichs-Lewy Condition}

Since a new flux is introduced into the equations, the CourantFriedrichs-Lewy (CFL) condition needs to be adjusted accordingly. In the context of an implicitly coupled ablation-flow code, the recession distance is imposed; the node velocity is therefore a function of the time step. This translates to

$$
\Delta t=\frac{l}{\sqrt{\left(u_{n}-\bar{w}_{n}\right)^{2}+u_{t}^{2}+u_{p}^{2}}+a}
$$

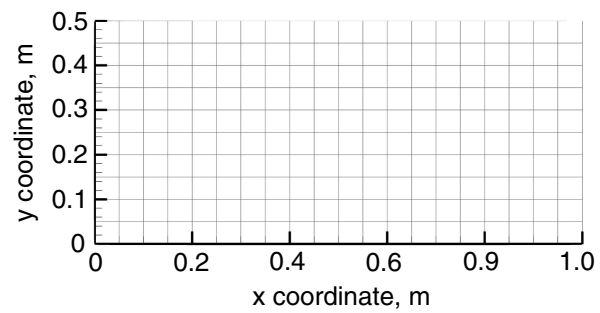

a) Original mesh
This equation can be solved for $\Delta t$ and yields

$$
\Delta t=\frac{l^{2}-l_{s}^{2}}{\left(l a-l_{s} u_{n}\right)+\sqrt{\left(l a-l_{s} u_{n}\right)^{2}-\left(l^{2}-l_{s}^{2}\right)\left(a^{2}-|u|^{2}\right)}}
$$

where $l$ is the characteristic length of the cell, $l_{s}=\frac{\Delta V}{S}$ is the characteristic length of the volume increment of the face, and $a$ is the speed of sound.

\section{Verification: Mass, Momentum, and Energy Conservation}

The first verification is performed on a simple geometry: a $0.5 \mathrm{~m} \times$ $1.0 \mathrm{~m}$ closed box, discretized with 10 cells $\times 20$ cells. The walls are adiabatic, and the gas (air), at rest, is initially at $15,000 \mathrm{~K}$, at a density of $0.1 \mathrm{~kg} / \mathrm{m}^{3}$. Because of chemistry, the temperature eventually relaxes to a lower, equilibrium value, although the total density remains the same (no change in physical volume). The mesh is moved randomly for 75 iterations; the initial and final meshes are shown in Fig. 5. Figure 6 presents the isovalues for translational temperature, total density, and velocity; to show the order of precision at which these quantities are computed, their theoretical values have been subtracted so that they all should be zero ( $\rho$ and $T$ are nondimensionalized). As shown in the figures, every one of them is zero, at machine precision.

\section{Verification: Shock Tube}

The next verification demonstrates that the time integration on the moving mesh is preserved and that the flux across the moving faces is computed correctly for a moving shock. The geometry consists of a $1 \times 0.5 \mathrm{~m}$ closed box containing air at a temperature of $350 \mathrm{~K}$; the left side has a density of $1 \mathrm{~kg} / \mathrm{m}^{3}$, and the right side is at $0.01 \mathrm{~kg} / \mathrm{m}^{3}$. The shock is simulated over a period of $2.0 \times 10^{-4} \mathrm{~s}$. The initial mesh consists of 200 squares $\times 100$ squares and moves randomly (random walk) every iteration for a maximum allowable distance of $5 \times 10^{-5} \mathrm{~m}$. The simulation is run implicitly, without solving the diffusion fluxes (therefore only solving Euler's equation), with a CFL value of 1 , so that the simulation is time accurate. Using the normal CFL condition, the solver uses 53 iterations to reach the final simulation time.

Figure 7 shows a closeup view of the state of the mesh after having been moved randomly 53 times; as can be seen, some nodes have moved considerably over the time period. Figure 8 compares the moving mesh solution to the same solution on a fixed grid. The results show excellent agreement, even for the temperature, a quantity usually very sensitive to compression effects. The largest discrepancy is at the shock front; this is easily explained by the fact that the cells resolving the shock have their front consistently moved back and forth, which, in the end, can lead to a small displacement in the shock position. Figure 9 shows the uniformity of the solution in the $y$ direction; it can be seen that the solution remains smooth and uniform, although multiple mesh movements have occurred.

\section{Note on Lagrangian Flux}

Other hypersonic flow solvers $[\underline{23}, \underline{24}]$ use moving meshes without compensating the volumetric increment by a Lagrangian flux. For instance, for shock tailoring purposes, the previous solution is simply projected on the new mesh. Since hypersonic solutions are almost always in steady state, at specific trajectory points, this does not affect

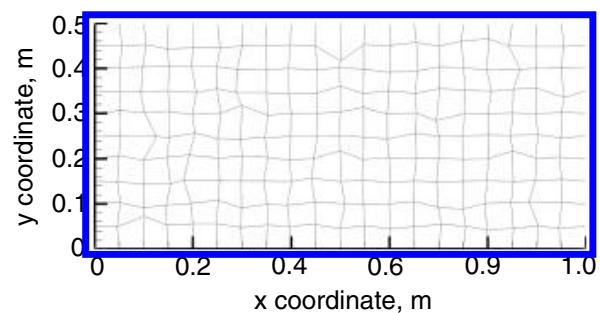

b) Final mesh

Fig. 5 Mesh movement for the first verification. 


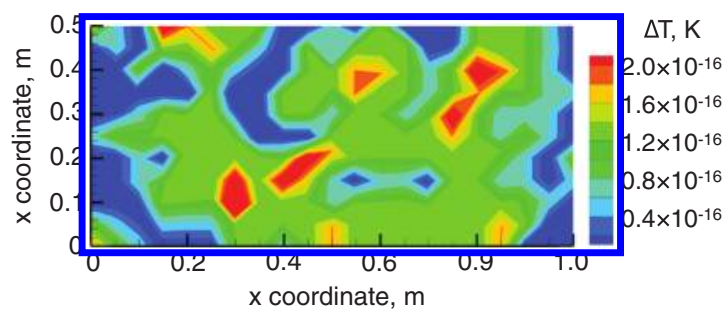

a) Temperature

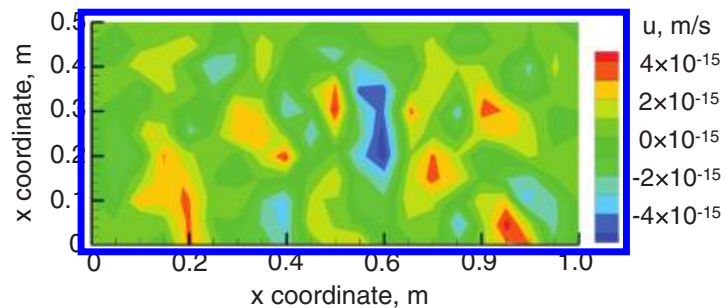

c) $x$ velocity

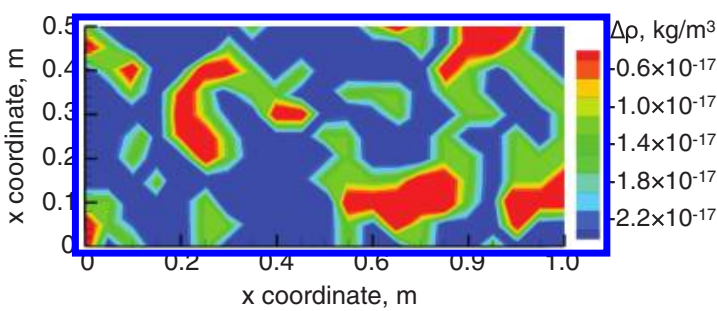

b) Density

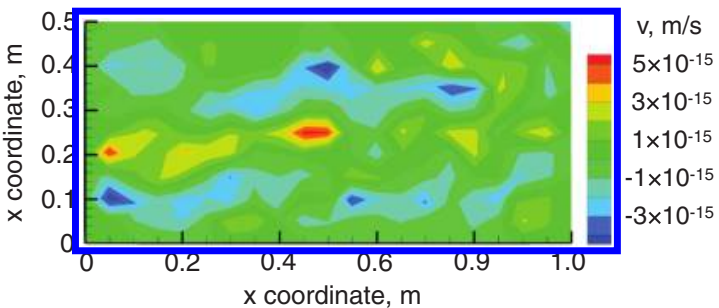

d) $y$ velocity

Fig. 6 Difference in computed and theoretical values after multiple random movements of the mesh.

too much the solution since only small mesh movements are performed, allowing the solution to relax and stabilize as the results converge. Considering that the Lagrangian fluxes are trivial to add to a flow solver, and that the volumetric increment needs to be computed anyway, the IGCL remains a more elegant and rigorous approach. And in the event of implementing mesh refinement through adaptation, these modifications would certainly prove to be useful. Moreover, and more importantly, the ALE approach opens up the possibility of using the coupling scheme for time-accurate problems.

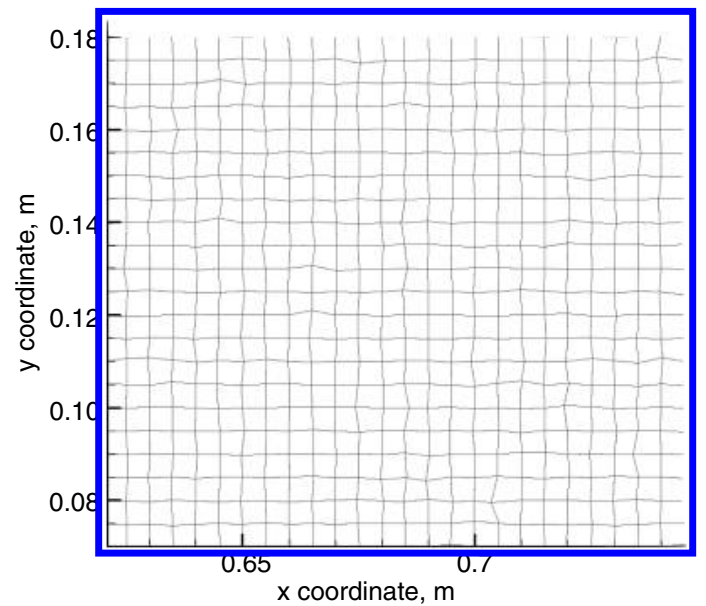

Fig. 7 Closeup view of the final mesh after 53 random movements.

\section{Mesh Movement Description}

\section{A. Surface Recession}

Even though the mathematics of the moving mesh is fairly simple, the question of how the mesh moves needs to be defined. In the context of ablation, because only the wall moves, the rest of the mesh can simply follow proportionally, using a perpendicular line between the wall and the inflow as guidance.

The algorithm first identifies the closest wall nodes for every node of the mesh. Then, a reference length is attributed to each wall node; this length corresponds to the distance between the wall node and the farthest mesh point attributed to that node. Each mesh node is then nondimensionalized according to these reference lengths. With this, each node has a coordinate system with a value ranging from 0 to 1 , distributed on a perpendicular line starting at the wall. This type of coordinate system is very similar to Landau coordinates.

Even though this method is far from being general, it is sufficient for the geometry usually studied in LeMANS. Complex geometries, especially if the surface is not physically uniform, would probably cause problems with this method. One way to generalize it would be to apply a smoothing scheme (averaging) to the relative coordinate in order to account for acute discrepancies in neighboring nodes.

\section{B. Shock Alignment}

As has been well established in the past $[\underline{25}, \underline{26}]$, one of the key conditions for obtaining accurate heat fluxes at the wall is the ability to resolve the shock properly. To do so, it is imperative to have the mesh as aligned as possible to the shock. Using a method defined in [24], the shock outer envelope is established by detecting the location where the Mach number takes the value of a fraction of the freestream

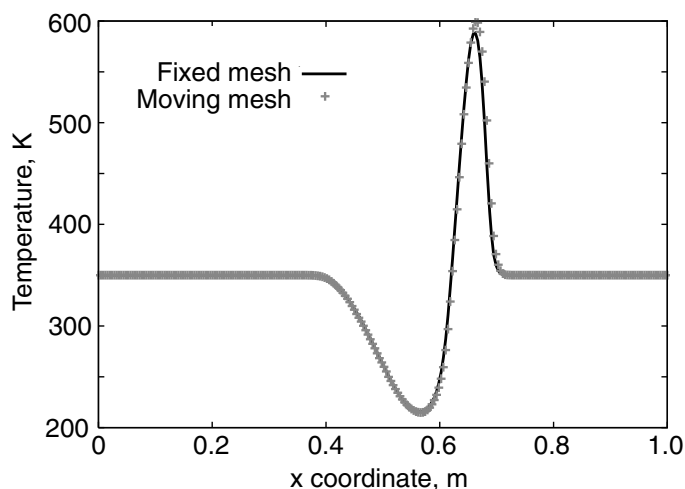

a) Temperature

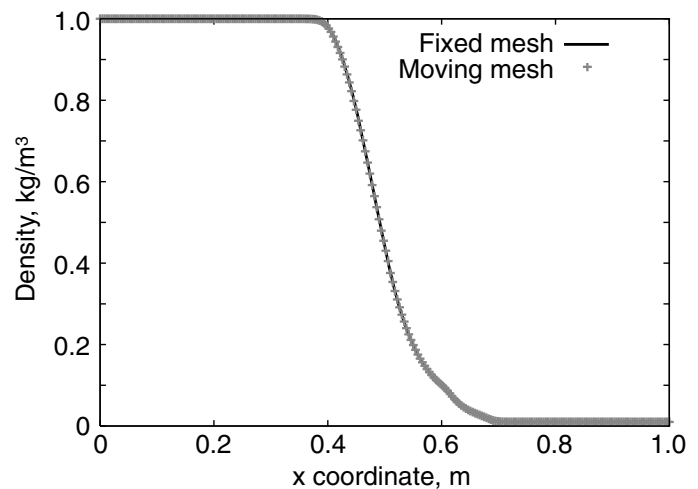

b) Density

Fig. 8 Comparison of the shock tube problem between a fixed mesh and a randomly moved mesh. 


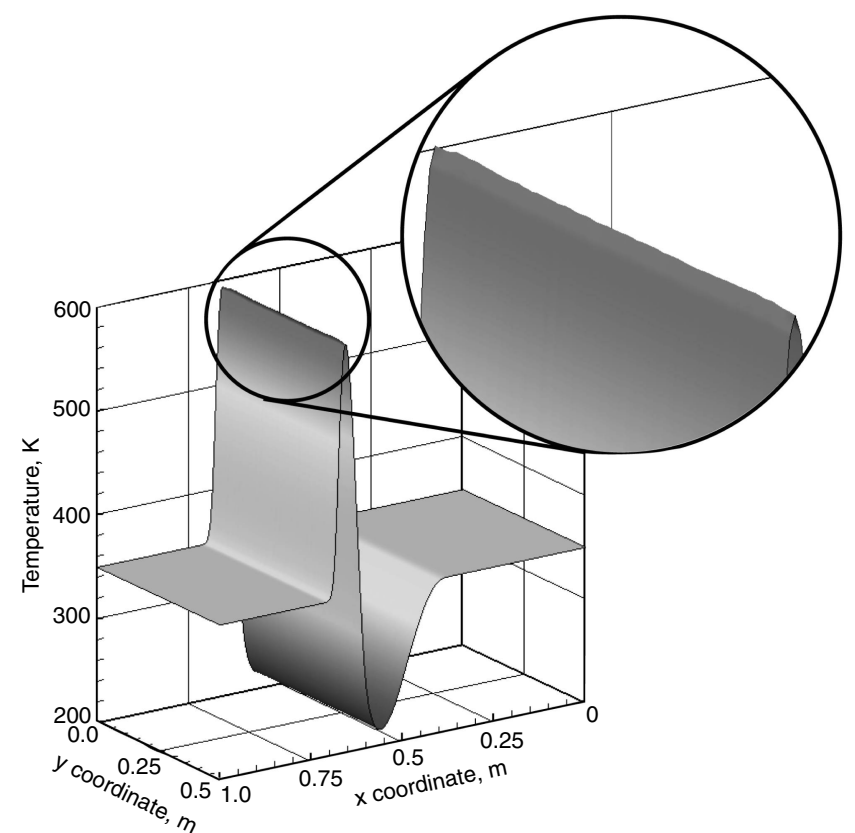

Fig. 9 Three-dimensional view of the shock tube solution using a moving mesh.

Mach number (this value is typically 99\%). Next, the new inlet boundary location is determined by offsetting the shock surface (or line, in two dimensions) by a constant normal distance. A fixed number of nodes is then imposed on lines perpendicular to the surface, between the shock surface and the new inlet surface, therefore insuring that the mesh faces remain aligned with the shock. The rest of the nodes are redistributed on the lines proportionally to their initial distribution. Optionally, a region of refinement can be added to properly capture the shock. This procedure is illustrated, in two dimensions, in Fig. 10a. For a simple test case, the resulting solution is presented in Fig. 10b, using isocontours of temperature.

Because of the ALE moving mesh capabilities described in the previous section, this procedure can be applied as the solution converges, without the need to stop and restart the simulation with an interpolated solution. This method also does not disrupt an already converged solution, although additional iterations must be allowed in order to compute the solution in the newly refined regions. Finally, as shown in Fig. 10, the method also offers the opportunity to refine the mesh in the shock region, to obtain a better solution.

\section{Strong Coupling Implementation}

Because reentry simulations are being performed by computing steady-state solutions at multiple points of a discretized trajectory, the

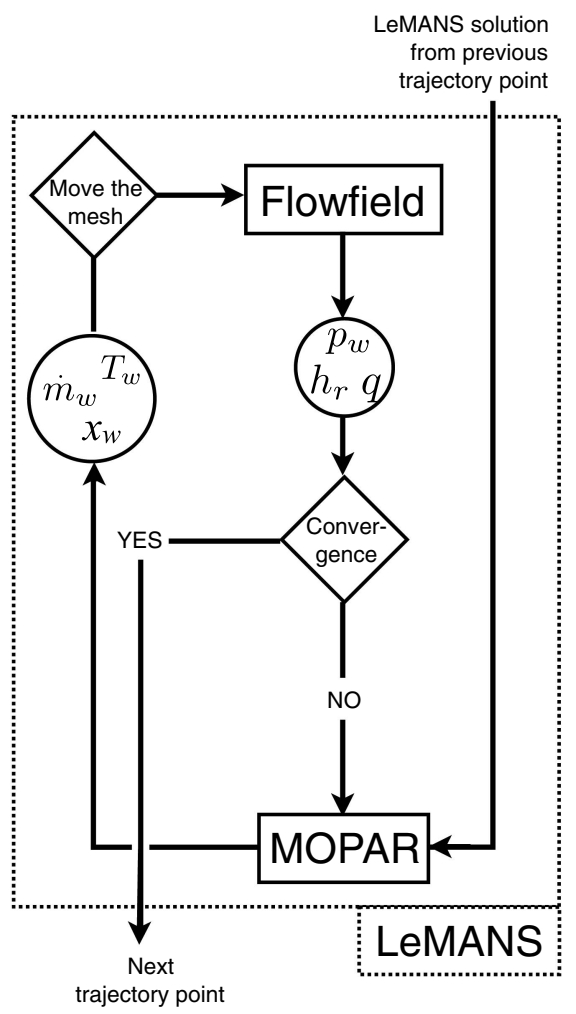

Fig. 11 Coupling procedure for the integration of MOPAR in LeMANS.

thermal response code is directly integrated as a boundary condition subroutine of LeMANS, thus taking advantage of the implicit nature of the code as well as the aggressive CFL ramping. The method, illustrated in Fig. 11, is similar to the one described in Kuntz et al. [27]

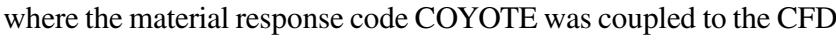
code SACCARA. The main difference resides in the fact that the coupling scheme of Kuntz et al. [27] does not use the ALE approach, and the mesh needs to be regenerated each time it is moved. Moreover, the mesh is not realigned to the shock as the freestream conditions change at various trajectory points. It is important to point out that COYOTE has not been designed to model a charring ablator, which greatly simplifies the problem for two-dimensional coupling.

Since MOPAR uses a one-dimensional formulation, normal solution lines within the wall are traced at each boundary cell and are computed sequentially. Because there is no need to compute the material response at every flowfield iteration, MOPAR is called at a predetermined number of iterations. This coupling method was previously presented [13], but without adapting the fluid mesh. Even though the method showed good results and proved to be efficient and

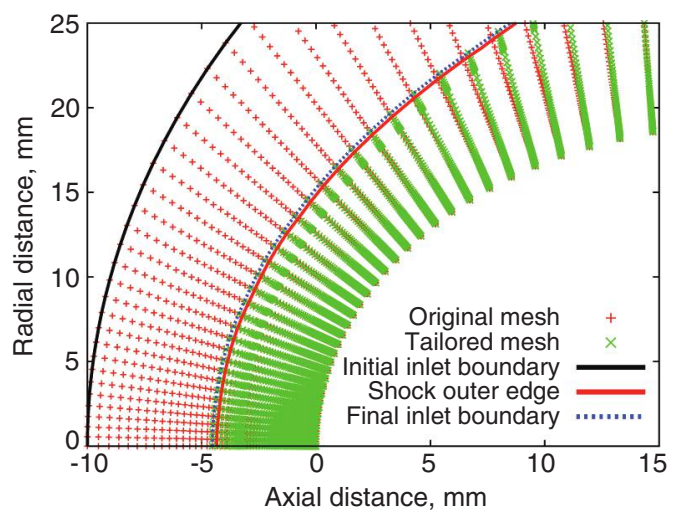

a) Initial and final mesh, with a front offset of $2 \times 10^{-4} \mathrm{~m}$ from the shock outer boundary

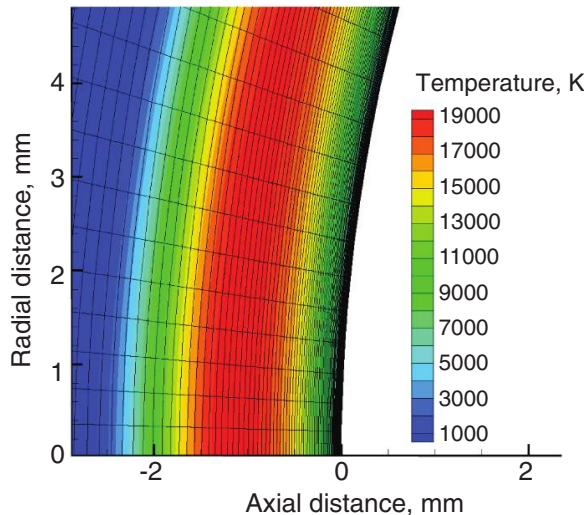

b) Example of a well-aligned shock obtained with the described procedure

Fig. 10 Mesh alignment procedure. 
Table 2 Freestream conditions for the reentry trajectory of the IRV-2 vehicle (from [27])

\begin{tabular}{lccccc}
\hline \hline $\begin{array}{l}\text { Trajectory } \\
\text { point }\end{array}$ & $\begin{array}{c}\text { Time, } \\
\mathrm{s}\end{array}$ & $\begin{array}{c}\text { Altitude, } \\
\mathrm{km}\end{array}$ & $\begin{array}{c}\text { Velocity, } \\
\mathrm{km} / \mathrm{s}\end{array}$ & $\begin{array}{c}\text { Temperature, } \\
\mathrm{K}\end{array}$ & $\begin{array}{c}\text { Density, } \\
\mathrm{kg} / \mathrm{m}^{3}\end{array}$ \\
\hline 1 & 0.00 & 66.9 & 6.78 & 228.0 & $1.25 \times 10^{-4}$ \\
2 & 4.25 & 55.8 & 6.79 & 258.0 & $5.05 \times 10^{-4}$ \\
3 & 6.75 & 49.3 & 6.79 & 271.0 & $1.13 \times 10^{-3}$ \\
4 & 8.75 & 44.0 & 6.77 & 261.0 & $2.26 \times 10^{-3}$ \\
5 & 10.25 & 40.1 & 6.75 & 250.0 & $4.00 \times 10^{-3}$ \\
6 & 11.50 & 36.8 & 6.72 & 242.0 & $6.43 \times 10^{-3}$ \\
7 & 12.50 & 34.2 & 6.68 & 234.0 & $9.58 \times 10^{-3}$ \\
8 & 13.25 & 32.3 & 6.64 & 229.0 & $1.31 \times 10^{-2}$ \\
9 & 13.95 & 30.5 & 6.60 & 227.0 & $1.73 \times 10^{-2}$ \\
\hline \hline
\end{tabular}

robust, the material response was not accurate since the shock wave was calculated from the initial state of the geometry, without taking into account the recession of the wall $x_{w}$.

Three modifications are applied at the interface between the two codes to preserve stability and accelerate convergence. It is to be noted that the convergence criterion in LeMANS is based on a $\ell^{2}$ norm evaluated on the total density changes in every cell of the mesh. First, the convective heat flux used in MOPAR is adjusted using a hotwall correction [28]:

$$
q_{h w}=q_{c w} \Omega_{h w}=q_{c w}\left[\frac{\rho\left(T_{h w}, p_{w}\right) \mu\left(T_{h w}\right)}{\rho\left(T_{c w}, p_{w}\right) \mu\left(T_{c w}\right)}\right]^{0.1}
$$

This method uses a boundary-layer approximation to estimate the heat flux and naturally disappears once the wall has reached its converged values (i.e., when $T_{c w}=T_{h w}$, then $q_{c w}=q_{h w}$ ). The use of this correction speeds up the convergence of the wall temperature and ablation rates and prevents the wall conditions from affecting significantly the convergence of the flowfield.

The second modification consists of damping the updated values at the wall. Instead of using the actual computed value given by the material response for recession distance, wall temperature, and blowing rates, the value is combined with the one computed at the previous iteration:

$$
T_{\text {assigned }}=(1-\psi) T_{\text {old }}+\psi T_{\text {computed }}
$$

The $\psi$ parameter, usually set to 0.75 , prevents the solution from being caught in an oscillation between two values and also prevents the values from being overevaluated (or under) while the solution is still changing. Other values were tested and led, in some case, to nonconvergence when values were too high or to a decoupled solution when the values were too low and failed to significantly impact the flowfield over the normal convergence period.

Figure 12a shows a comparison between the convergence history of a simulation (the IRV-2 vehicle, formally presented in the following section), using a reradiating wall temperature boundary condition, the coupled method with no moving mesh, and the coupling method with the moving mesh. The simulation uses a ramping CFL number, with initial conditions set to the converged solution at the previous trajectory point, using their respective wall temperature boundary. For this case, MOPAR is called every 100 iterations. The first observation to make is that it is impossible to see where MOPAR is called on the nonmoving mesh curves for this particular simulation; this is explained by the fact that the residual $\ell^{2}$-norm at the wall

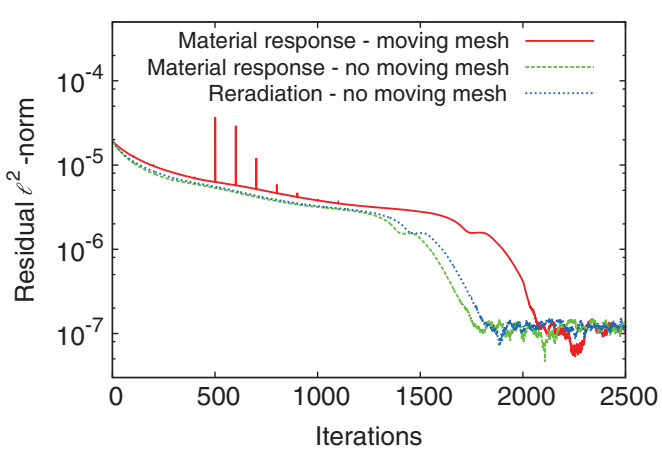

a) Function of the number of iterations

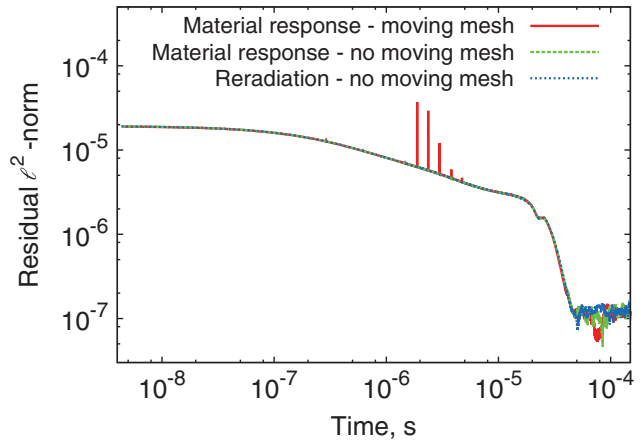

b) Function of the physical time

Fig. 12 Convergence to steady state for the IRV-2 vehicle, at trajectory point 2 of Table 2: comparison between the regular solution and the coupled method.

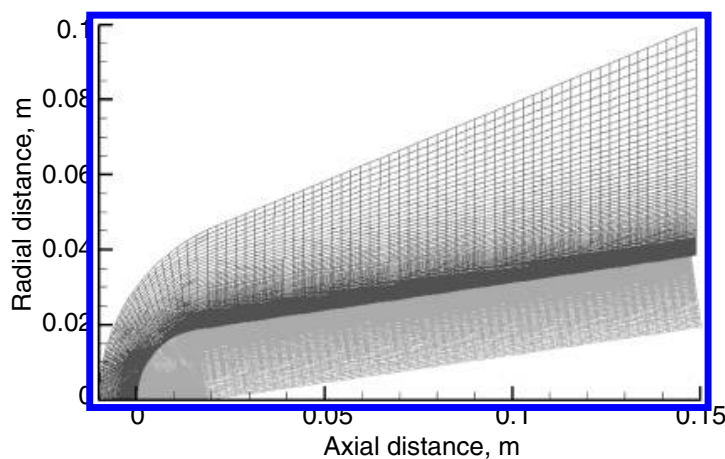

a) Overall view of the meshes

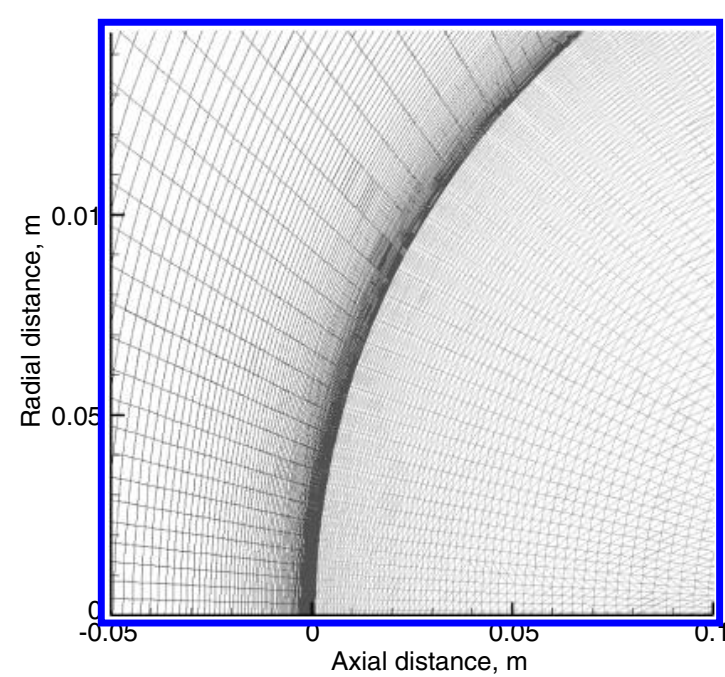

b) Closeup view of the meshes

Fig. 13 Flowfield mesh and material response mesh used for the simulation of the reentry of the IRV-2 vehicle, at trajectory point 2 of Table 2. 


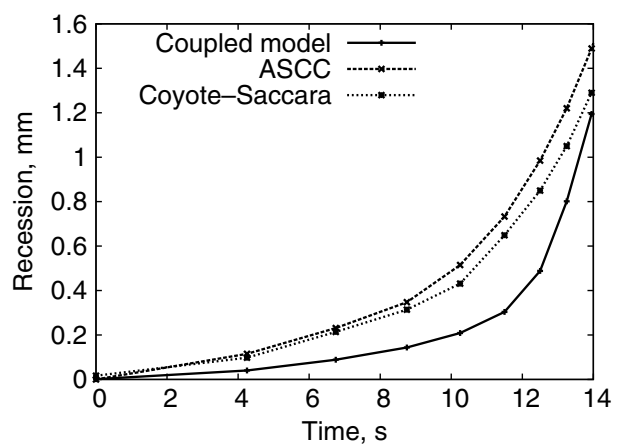

a) Surface recession

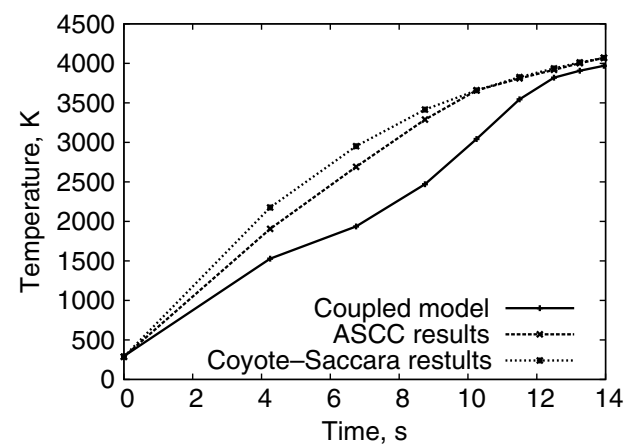

b) Surface temperature

Fig. 14 Comparison of surface recession and surface temperature at the stagnation point during reentry for the IRV-2 vehicle.

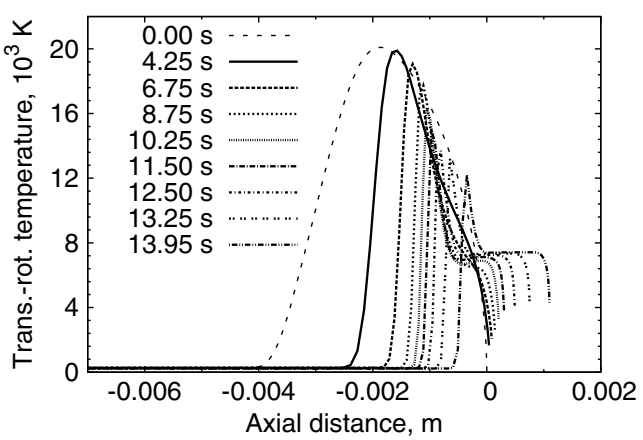

a) Solution without shock tailoring

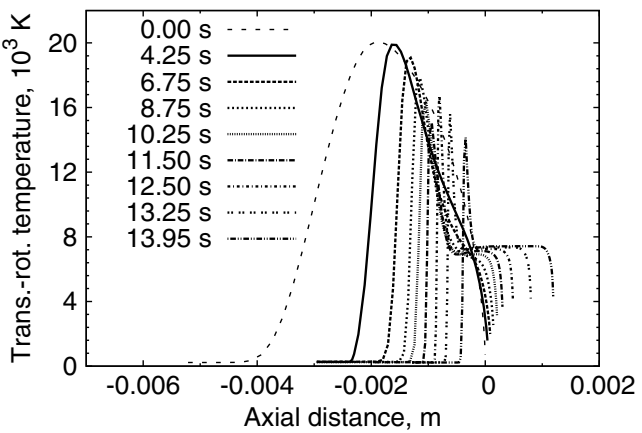

b) Solution with shock tailoring

Fig. 15 Translational-rotational (Trans-rot) temperature along the stagnation line at the different trajectory points.

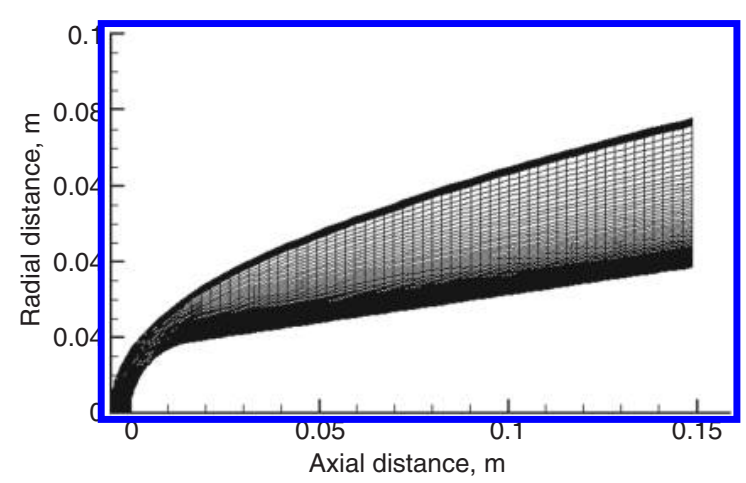

a) Tailored mesh

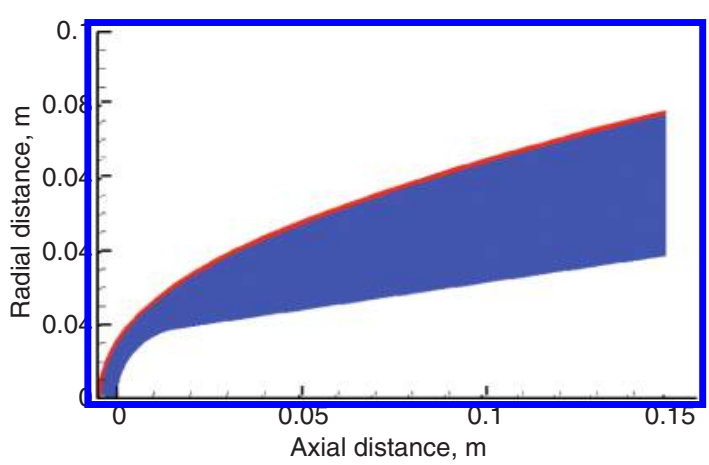

b) Mach isocontour: the red region has a value of more than $99 \%$ of the freestream mach number, and the blue region has a value of less

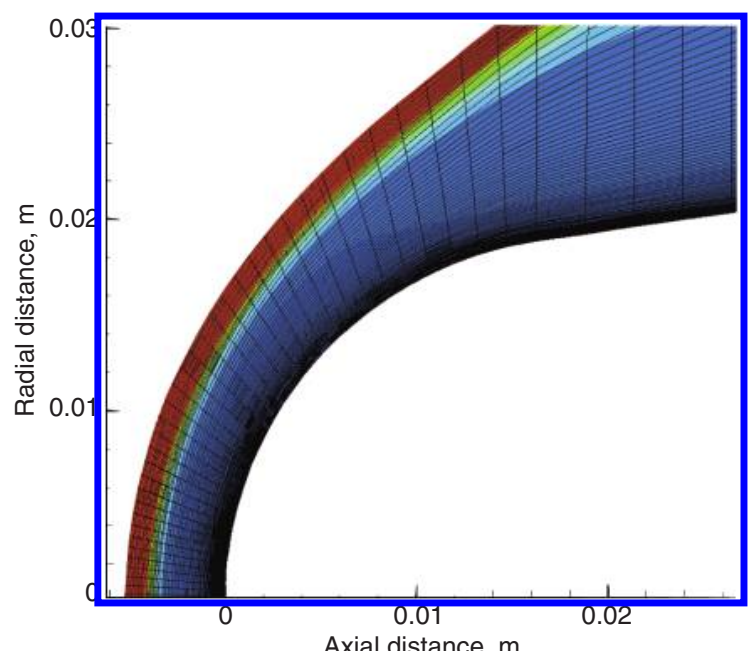

c) Mach isocontours, with the cells aligned

Fig. 16 Adapted mesh for the second trajectory point. 


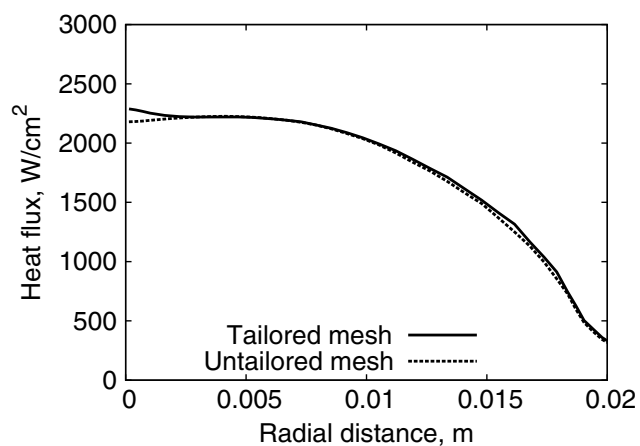

a) Surface heat flux

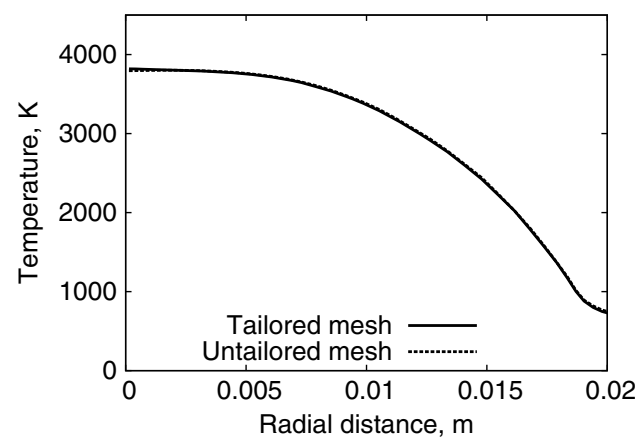

c) Surface temperature

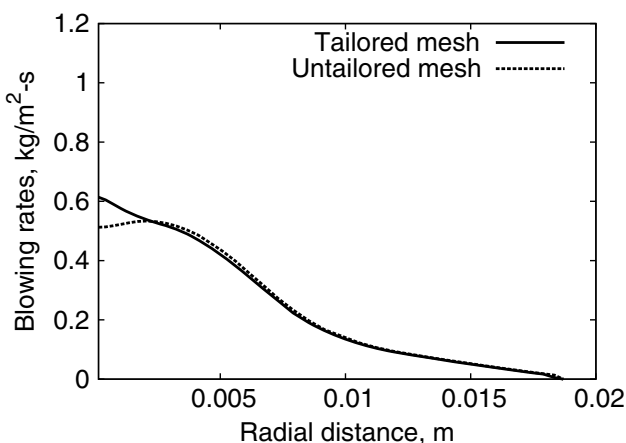

b) Surface blowing rate

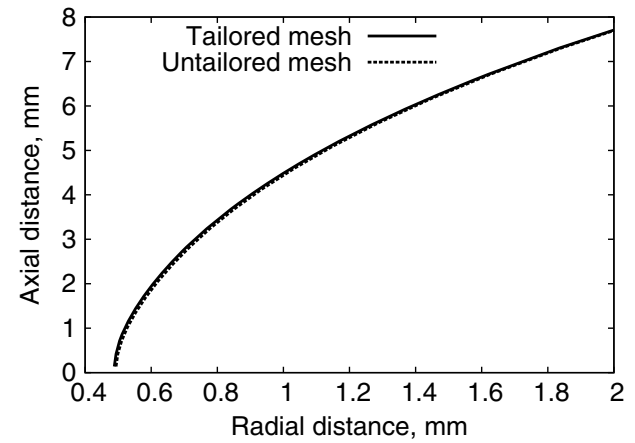

d) Surface recession

Fig. 17 Improvement of the solution using mesh tailoring, for trajectory point 7, at $t=12.50 \mathrm{~s}$.

is always smaller than in the rest of the flowfield. It is, however, quite different for the moving mesh curve: spikes can be seen in the curves each time the material response code is called. Those spikes are caused by the fact that the residual $\ell^{2}$-norm is calculated using the right-hand side of Eq. (22); however, using this, the volume increment due to the moving mesh is not taken into account and causes the spikes in the convergence curves. The addition of a term in the residual $\ell^{2}$-norm calculation would therefore remove the spikes; however, they can give a good indicator of the degree of mesh movement. Using them, it is possible to see when the freestream flow hits the wall (around 500 iterations) as well as the effects this sudden impact has on the wall conditions. It can also be seen on Fig. 12a that the residual errors induced by the mesh movements quickly become smaller than the usual, convergence residuals; after roughly 1000 iterations, the spikes are no longer visible on the plots.

The three curves plotted in Fig. 12a follow the same trend and show a similar convergence level. In fact, as shown in Fig. 12b, these curves are effectively identical when plotted against the physical time

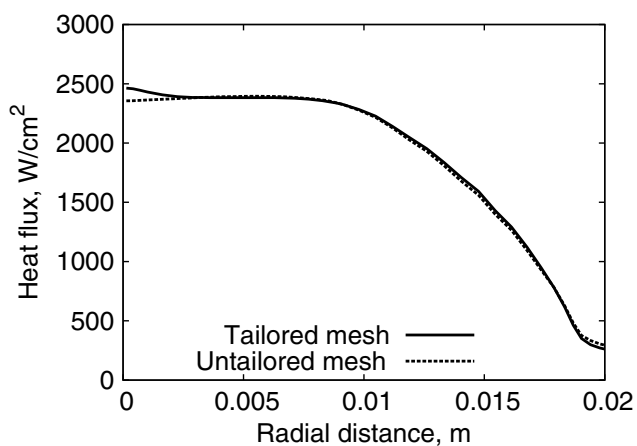

a) Surface heat flux

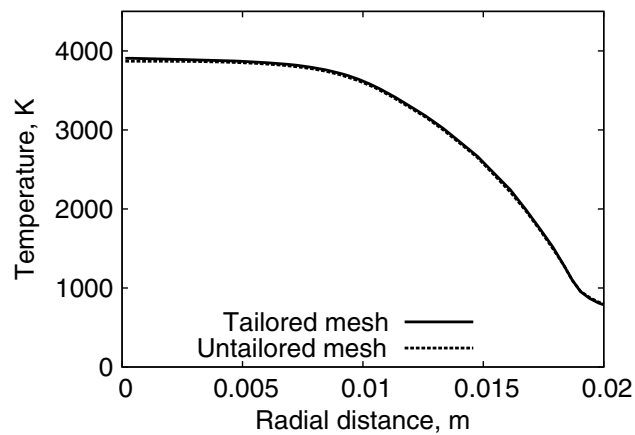

c) Surface temperature

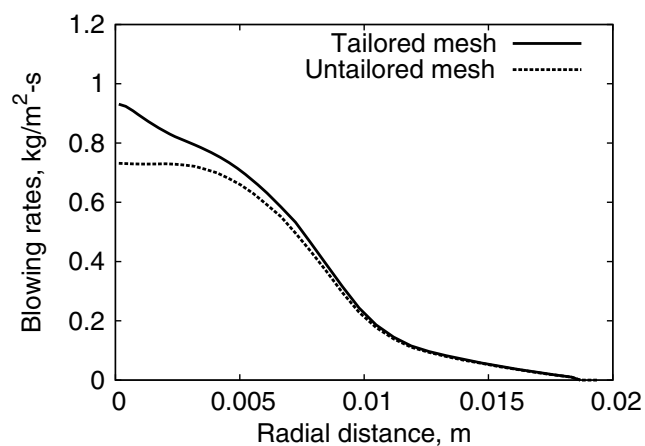

b) Surface blowing rate

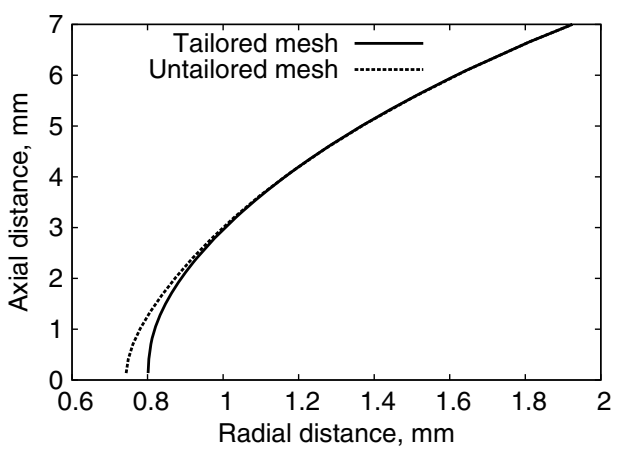

d) Surface recession

Fig. 18 Improvement of the solution using mesh tailoring, for trajectory point 8, at $t=13.25 \mathrm{~s}$. 


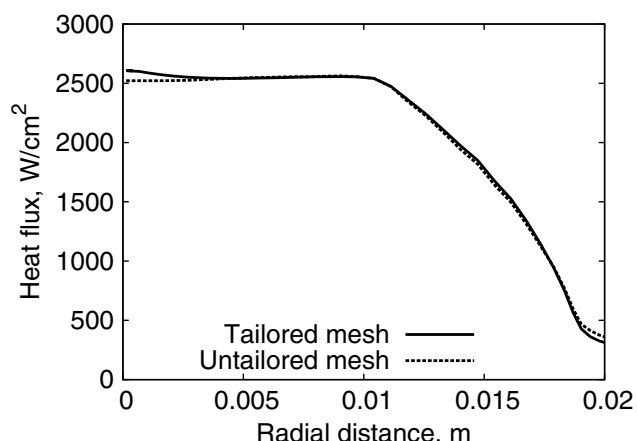

a) Surface heat flux

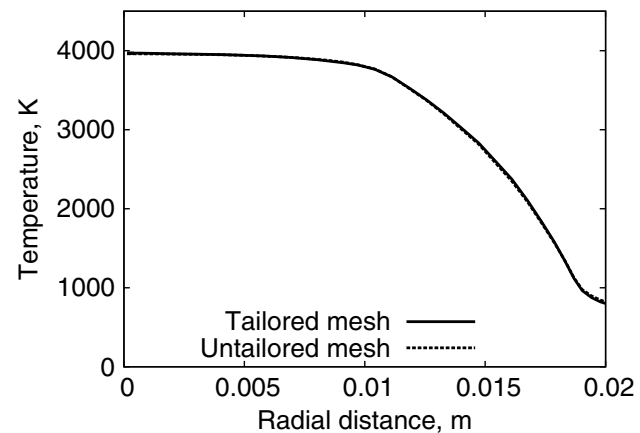

c) Surface temperature

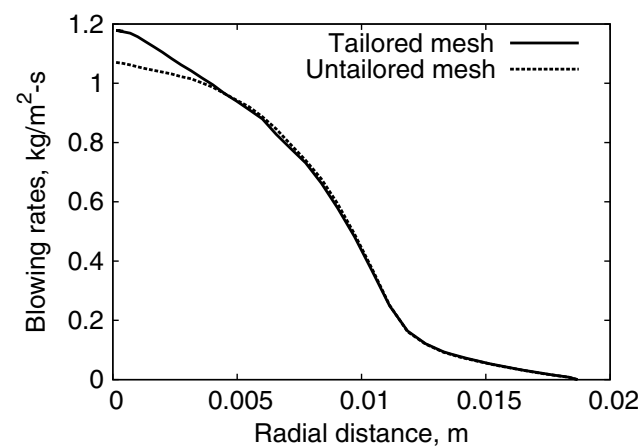

b) Surface blowing rate

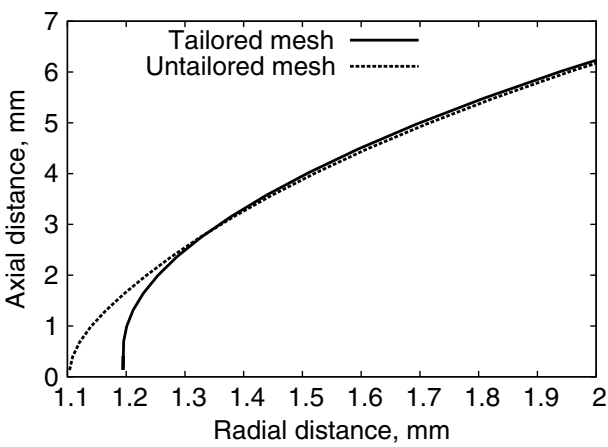

d) Surface recession

Fig. 19 Improvement of the solution using mesh tailoring, for trajectory point 9, at $t=13.95 \mathrm{~s}$.

instead of the number of iterations. Considering that the highest source of error (residual) is from the flowfield, and not wall coupling, this is not surprising. The difference in time steps is caused by a combination of the additional fluxes and different cell sizes due to the mesh movement. Therefore, even though the wall clock time is obviously greater (around 10\% in this particular test case), the presented coupling method does not affect the physical time of convergence nor the robustness of the solution.

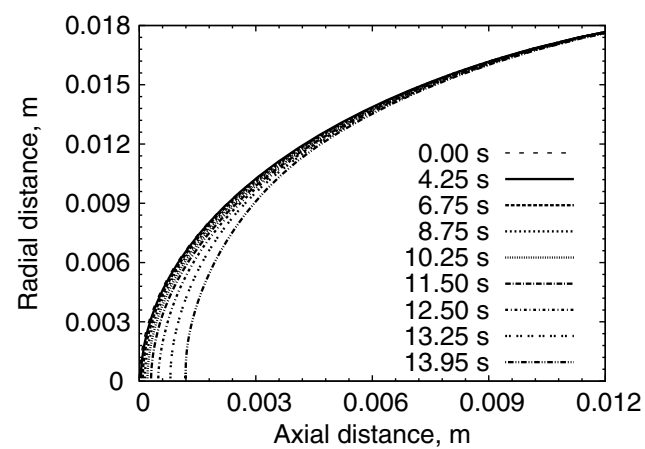

a) Surface recession

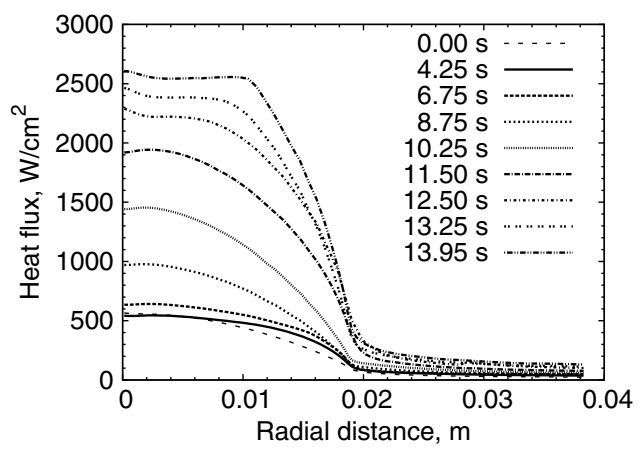

c) Heat flux at the surface
Finally, the method used to couple the recession distance must be discussed. Because MOPAR is employed in each cell neighboring the wall, the recession distance is therefore calculated at the face of the cell. However, the moving mesh scheme presented here uses node velocities to move the wall (and the rest of the cells). Therefore, the face recession distance must be transformed into node velocities. To do so, the displacement of each node is taken as an average of each of its neighboring faces and then divided by the time step to obtain a

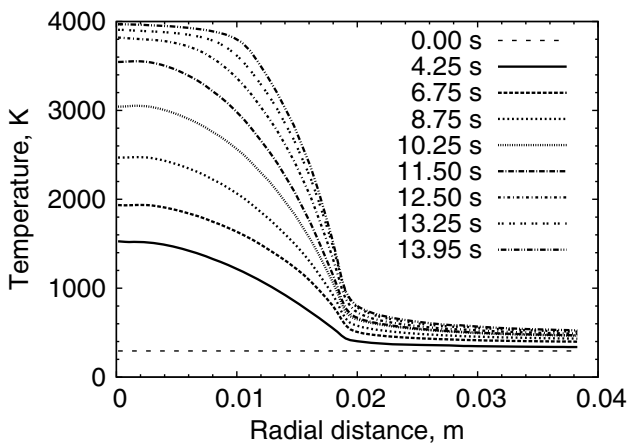

b) Surface temperature

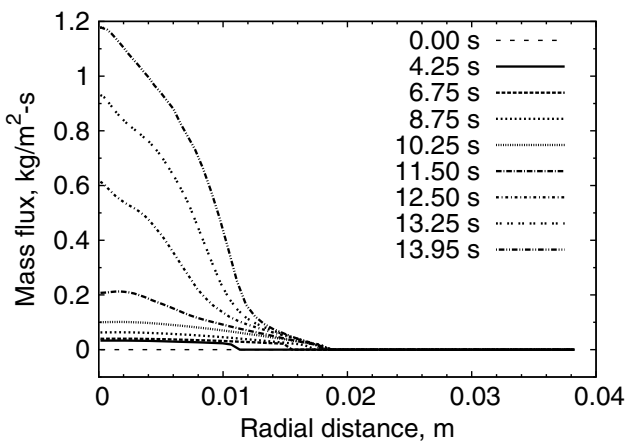

d) Blowing rates at the surface

Fig. 20 Surface properties during reentry for the IRV-2 vehicle, using the mesh tailoring algorithm. 
velocity. The number of mesh point at the wall interface is assumed to be sufficient for the averaging method to be accurate. Therefore, the wall-interface remains smooth throughout the simulation.

It is to be noted that the only reason recovery enthalpy $h_{r}$ is needed in this coupling scheme is because surface thermochemical tables (the so-called $B^{\prime}$ tables) are used by MOPAR to evaluate the ablation rates. The recovery enthalpy is used to transform the dimensionless parameter $B^{\prime}$ into an actual blowing rate, as shown in Eqs. (18) and (19). If those tables were not needed, for instance, if a surface kinetic module were used [29], $h_{r}$ would not be needed.

\section{Results: IRV-2 Test Case}

To demonstrate the strong coupling between MOPAR and LeMANS, the well-documented [17,27] reentry simulation of an IRV-2 vehicle is performed. The freestream conditions used in the discretized trajectory are presented in Table $\underline{2}$, and the material properties are set to generic noncharring carbon, using the properties given by [30]. The ablation rates are interpolated from thermochemical tables generated by ACE-SNL [31] for carbon in air. Reradiation is also included at the boundary. The initial mesh used for the simulation is presented in Fig. 13; it is important to point out that the material response calculations are carried out in one dimension, as described earlier: the triangular mesh presented in the figure is generated for postprocessing analysis. The flowfield mesh consists of $88 \times 100$ nodes, and the solid mesh is composed of 88 lines of 75 nodes each. A mesh refinement study was performed on the initial mesh, and those values were chosen because they provide a reliable solution in a reasonable time.

Because the purpose of this test case is to demonstrate the coupling scheme, the flowfield simply uses Park's [32] five-species finite-rate chemistry model for air. Carbon chemistry in the flowfield is thus
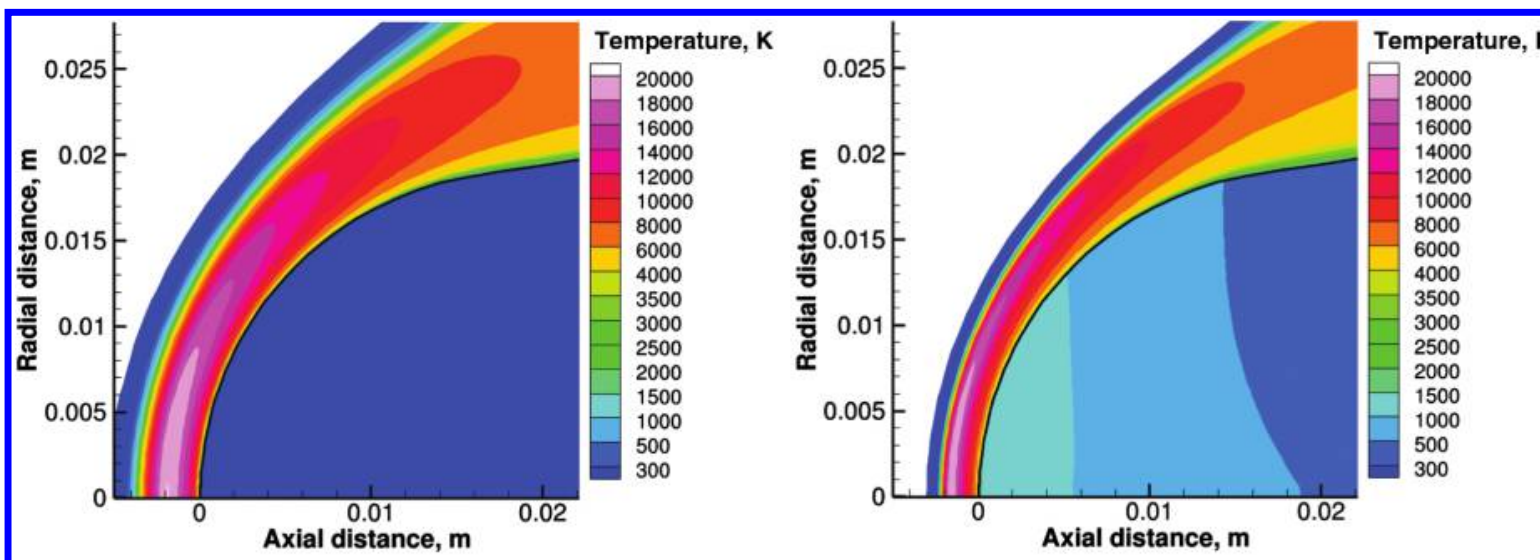

a) $t=0.00 \mathrm{~s}$

b) $t=4.25 \mathrm{~s}$
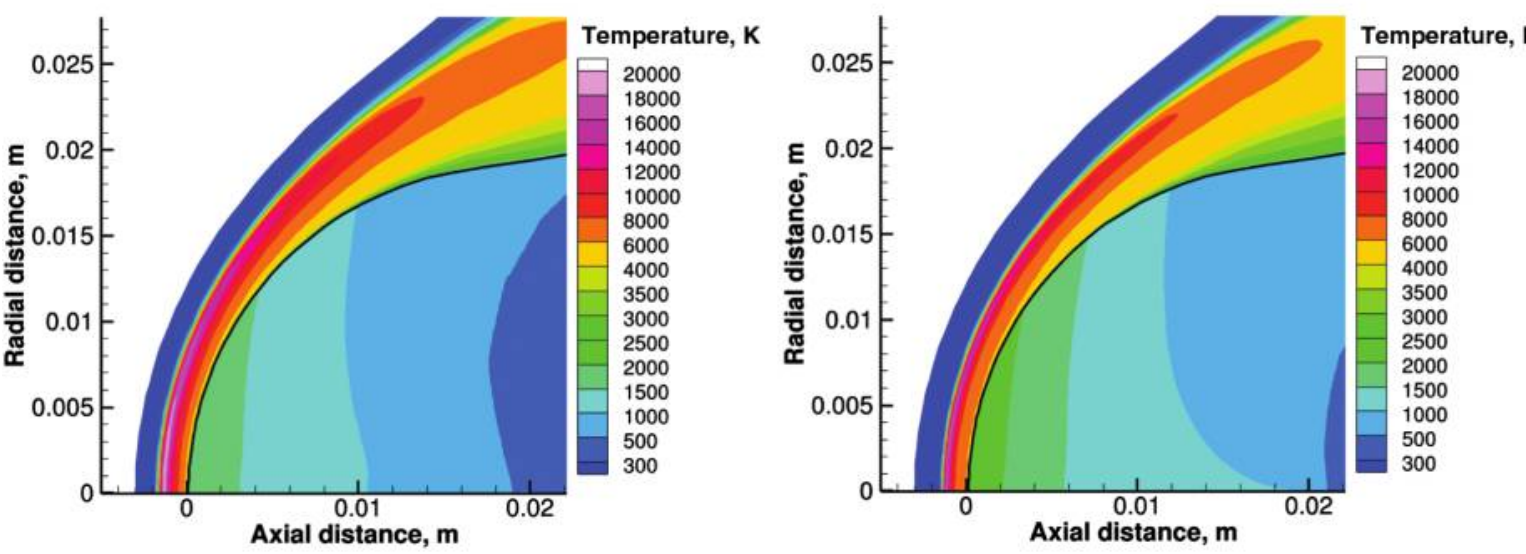

c) $t=6.75 \mathrm{~s}$

d) $t=8.75 \mathrm{~s}$
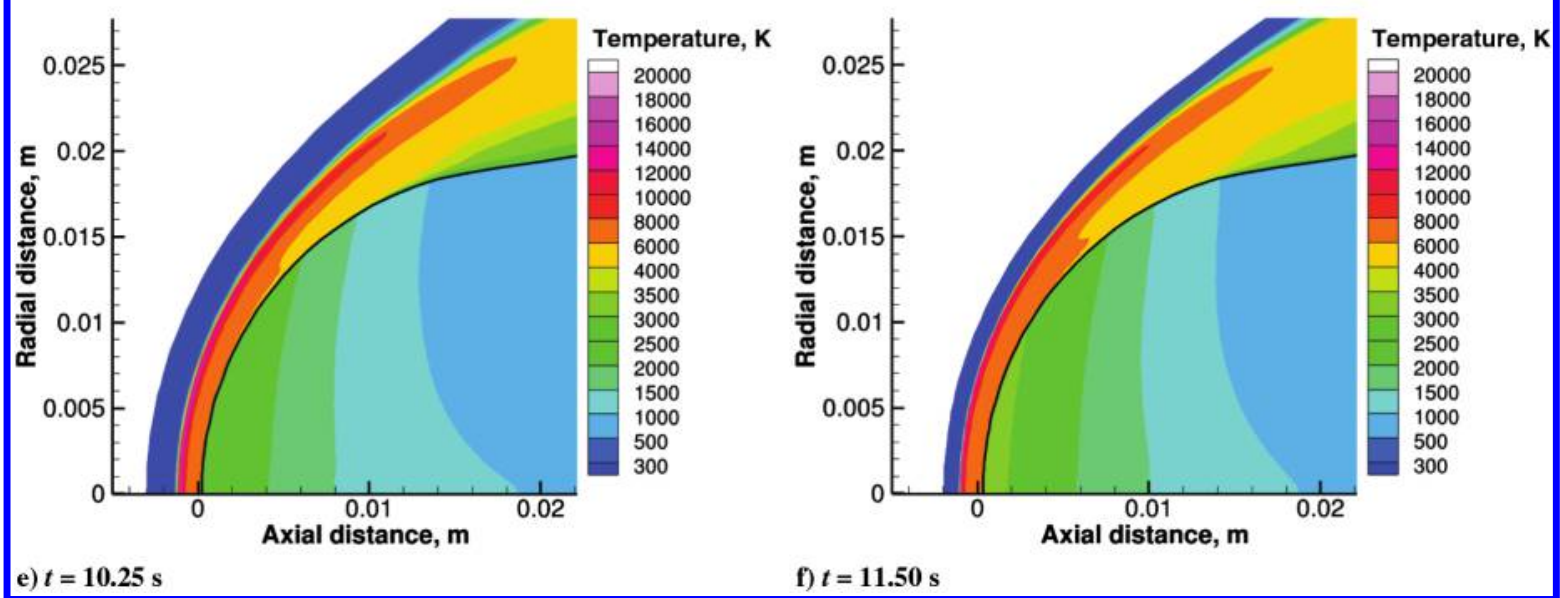

e) $t=10.25 \mathrm{~s}$

f) $t=11.50 \mathrm{~s}$

Fig. 21 Translational temperature of the flowfield and temperature in the solid wall of the IRV-2 reentry vehicle at trajectory points 1 through 6 of Table 2 . 
omitted from this simulation. Although the density at the wall accounts for ablation [Eq. (12)], the species composition remains the same as the one computed by the flowfield with a noncatalytic boundary condition.

The results for the recession distance and surface temperature at the stagnation point over the reentry trajectory are shown in Fig. 14. The computed values are compared to results obtained with the $\overline{A S C C}$ code, which includes flight data, and with the coupled COYOTE-SACCARA codes [27]. As can be seen, the results are within the expected range, considering that thermodynamic values and ablation rates for a generic carbon-carbon ablator are used (the ablator properties are not reported in the study). Moreover, the computed results neglect carbon chemistry in the flowfield, thus neglecting chemical reactions in the boundary layer, as well as the contribution of those species to the diffusive heat flux at the surface. Considering that a noncatalytic boundary condition is applied, it is therefore not surprising that the heat flux and thus the recession distance and temperature are underestimated (a noncatalytic boundary layer is considered to be the lower bound when used as a simplified wall kinetic model). For this particular example, the coupled algorithm proved to be fast and robust for all the trajectory points (the results were obtained in roughly $18 \mathrm{~h}$, using 32 processors).

The translation temperature sampled on the stagnation streamline is presented in Fig. 15, for both the solution with shock tailoring and without. It shows how the shock evolves, both in location and in shape, as the vehicle enters the atmosphere. From this figure, it is quite evident that the mesh refinement requirements must vary from one trajectory point to another. For the results without shock tailoring, Fig. 15a, it can be seen that as time increases the section of the domain located in front of the shock increases, and therefore more and more mesh cells end up in the freestream region where all the calculated properties are invariant. This also has the side effect of reducing the resolution of the mesh, which then becomes inadequate in the vicinity of the shock. For instance, for the last trajectory point $(t=13.95 \mathrm{~s})$, the structure of the shock is resolved using approximately 7 mesh cells, and more than $40 \%$ of the cells are in the freestream region. Additional inaccuracies are caused by the fact that the nodes are only allowed to move on fixed lines in the flowfield. The perpendicularity to the surface is affected as the surface recesses, therefore deteriorating the solution on the adjacent cells. Also, as the freestream conditions and surface geometry change, the lack of a mesh quality verification algorithm causes the mesh to become misaligned with the shock, which causes errors in the heat flux at the surface. In a strongly coupled scheme, these kinds of errors grow over time since an imprecision in one solution is ported and amplified at each subsequent trajectory point. This is especially true for sensitive quantities such as heat flux. On top of these mesh quality problems, it is fair to assume that an approximate shock resolution will have effects on the nonequilibrium chemistry calculations since the temperatures are not evaluated properly. A similar claim could be made about the nonequilibrium radiation calculations, which might be significant in certain reentry trajectories, for certain vehicle geometries.

Figure $15 \mathrm{~b}$ shows the effect of refining the mesh by tailoring it to the shock and redistributing the cells in the region of high gradients. When compared to the untailored solution in Fig. 15a, it can be seen that the untailored approach produces a wider shock, with a much lower peak temperature. One of the adapted meshes is shown in Fig. 16a, and the isocontour used for delimiting the edge of the shock (99\% of the freestream Mach Number) is shown in Fig. 16b. A

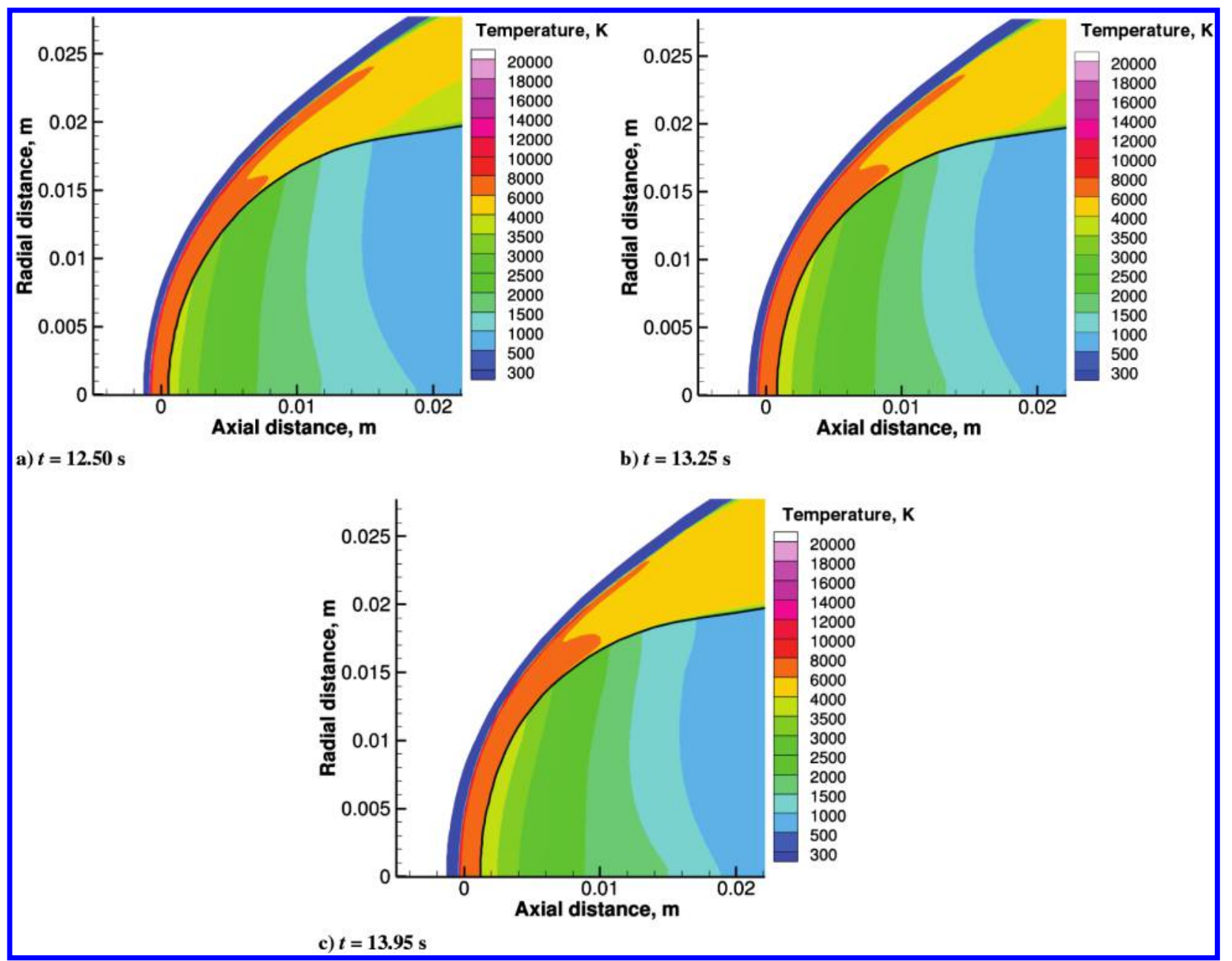

Fig. 22 Translational temperature of the flowfield and temperature in the solid wall of the IRV-2 reentry vehicle at trajectory points 7 through 9 of Table 2 . 
distance of $1 \mathrm{~mm}$ has been chosen to precede the edge of the shock. Figure 16c shows a closeup view of the cells aligning with the Mach number isocontours.

Figures 17-19 show a comparison between the tailored and untailored results for trajectory points 7, 8, and 9. As can be seen, the effects are significant, and the heat fluxes and blowing rates have the expected shape at the stagnation point (for an axisymmetric geometry). More importantly, the off-axis dip in the untailored solution, usually generated by shock misalignment, disappears and is not ported to the subsequent trajectory points. As for the blowing rates, the difference is quite important between the two solutions, with differences nearing 30\%. The blowing rate is an important parameter as it modifies the boundary-layer structure through momentum injection [33]. It also influences the boundary-layer chemistry, which in turn influences the surface heat flux [34]. It is to be noted that the benefits of mesh tailoring are less noticeable when looking at temperature since at the surface it reaches a maximum value regardless of the incoming heat flux (sometimes called temperature of ablation). For that reason, and because the present test case does not model a charring ablator, the difference in the heat fluxes transposes directly to a difference in the blowing rates.

The overall results for the trajectory of Table 2 are presented in Fig. 20. The evolution of the solution looks smooth and uniform for all surface properties. Finally, the carbuncle phenomenon was never seen in any steps of the solution, most likely because of the high diffusivity of the Steger-Warming flux vector splitting scheme.

Figures 21 and 22 present the isocontours of temperature in the flowfield as well as within the material. As can be seen, the surface remains smooth even though the coupling is aggressive, as the vehicle ablates toward its final shape. It is also important to note that, even though the mesh is relatively sparse at the wall in the tangential direction, the face-to-node interpolation remains very accurate.

\section{Conclusions}

To improve heat and ablation rate modeling on hypersonic reentry vehicles, a material response code was strongly coupled to a hypersonic flow solver. Verifications of the blowing boundary condition were presented, using two semi-analytical cases. The first, based on the Blasius solution of the boundary-layer problem, showed excellent agreement with the simulation results. The second, based on Kays and Crawford's blowing correction, also produced good results; the previously discussed problem with the fitting parameter $\lambda$ was also observed.

Verification of a moving mesh algorithm was also presented, using two test cases. The first one insured that no mass, energy, or momentum was created when the mesh was aggressively moved, and the second validated that the mesh movement did not change the ability of the code to capture moving shocks. For both cases, the results were well within the expected range, showing machine precision errors. These tests also showed that the complex nonequilibrium and chemistry source terms as well as the very stiff fluxes were unaffected by the moving mesh. A mesh tailoring procedure was also presented. This technique, applied as the solution converged, prevented the results from deteriorating as the shape of the vehicle and freestream condition changed and causing the mesh to become misaligned with the shock.

Finally, to demonstrate the coupling between a flow solver and a material response code, the simulation of the initial phase of the reentry trajectory of the IRV-2 vehicle was presented. The numerical results were compared with published results that used flight data. The comparison showed that the computed results were within a reasonable range given the numerous assumptions.

The coupling method presented here showed stability, robustness, and efficiency when used to coupled difficult problems. But more importantly, it provided the possibility of improving TPS modeling by providing a framework that will allow the removal of most of the surface balance inaccuracies introduced by the use of boundary-layer assumptions, heat and mass transfer coefficients, and equilibrium thermochemical tables.

\section{Acknowledgments}

The authors would like to thank the Government of Québec, which, through the Fonds de Recherche sur la Nature et les Technologies, provided a fellowship to A. Martin. Additional funding was provided by NASA SBIR Phase-2 Award NNX10CC53P and NASA EPSCoR RA Award NNX13AN04A. I. D. Boyd also gratefully acknowledges funding for this work through Air Force Office of Scientific Research grant FA-9550-11-1-0309.

\section{References}

[1] Kinney, D. J., "Aerothermal Anchoring of CBAero Using High Fidelity CFD," AIAA Paper 2007-0608, 2007. doi:10.2514/6.2007-608

[2] Olynick, D., Chen, Y.-K., and Tauber, M. E., "Forebody TPS Sizing with Radiation and Ablation for the Stardust Sample Return Capsule," AIAA Paper 1997-2474, 1997.

[3] Martin, A., and Boyd, I. D., "Non-Darcian Behavior of Pyrolysis Gas in a Thermal Protection System," Journal of Thermophysics and Heat Transfer, Vol. 24, No. 1, 2010, pp. 60-68. doi: $10.2514 / 1.44103$

[4] Martin, A., Scalabrin, L. C., and Boyd, I. D., "High Performance Modeling of an Atmospheric Re-Entry Vehicle," Journal of Physics: Conference Series, Vol. 341, No. 1, 2012, Article 012002. doi:10.1088/1742-6596/341/1/012002

[5] Farbar, E. D., Boyd, I. D., and Martin, A., "Numerical Prediction of Hypersonic Flow Fields Including Effects of Electron Translational Nonequilibrium," Journal of Thermophysics and Heat Transfer, Vol. 27, No. 4, 2013, pp. 593-606. doi:10.2514/1.T3963

- [6] Wright, M. J., Candler, G. V., and Bose, D., "Data-Parallel Line Relaxation Method for the Navier-Stokes Equations," AIAA Journal, Vol. 36, No. 9, 1998, pp. 1603-1609. doi: $10.2514 / 2.586$

[7] Gnoffo, P. A., "Upwind-Biased, Point-implicit Relaxation Strategies for Viscous Hypersonic Flows," AIAA Paper 1989-1972-CP, 1989. doi:10.2514/6.1989-1972

[8] Nompelis, I., Drayna, T. W., and Candler, G. V., "A Parallel Unstructured Implicit Solver for Hypersonic Reacting Flow Simulation," AIAA Paper 2005-4867, 2005. doi: $10.2514 / 6.2005-4867$

[9] Scalabrin, L. C., "Numerical Simulation of Weakly Ionized Hypersonic Flow Over Reentry Capsules," Ph.D. Thesis, Univ. of Michigan, Ann Arbor, MI, 2007.

[10] Blackwell, B. F., and Hogan, R. E., "One-Dimensional Ablation Using Landau Transformation and Finite Control Volume Procedure," Journal of Thermophysics and Heat Transfer, Vol. 8, No. 2, 1994, pp. 282-287. doi: $10.2514 / 3.535$

[11] Amar, A. J., Blackwell, B. F., and Edwards, J. R., "One-Dimensional Ablation Using a Full Newton's Method and Finite Control Volume Procedure," Journal of Thermophysics and Heat Transfer, Vol. 22, No. 1, 2008, pp. 71-82. doi: $10.2514 / 1.29610$

[12] Amar, A. J., Blackwell, B. F., and Edwards, J. R., "Development and Verification of a One-Dimensional Ablation Code Including Pyrolysis Gas Flow," Journal of Thermophysics and Heat Transfer, Vol. 23, No. 1, 2009, pp. 59-71. doi: $10.2514 / 1.36882$

[13] Martin, A., and Boyd, I. D., "Simulation of Pyrolysis Gas Within a Thermal Protection System," AIAA Paper 2008-3805, 2008. doi:10.2514/6.2008-3805

[14] Martin, A., and Boyd, I. D., "Variant of the Thomas Algorithm for Opposed-Border Tridiagonal Systems of Linear Equations," International Journal for Numerical Methods in Biomedical Engineering, Vol. 26, No. 6, 2008, pp. 752-759. doi: $10.1002 / \mathrm{cnm} .1172$

[15] Lachaud, J., Martin, A., Cozmuta, I., and Laub, B., "Ablation Test-Case Series 1," 4th AFOSR/SNL/NASA Ablation Workshop, 2010.

[16] Lachaud, J., Martin, A., Cozmuta, I., and Laub, B., "Ablation Test-Case Series 2," 4th AFOSR/SNL/NASA Ablation Workshop, 2010.

[17] Thompson, R. A., and Gnoffo, P. A., "Implementation of a Blowing Boundary Condition in the LAURA Code," AIAA Paper 2008-1243, 2008.

doi: $10.2514 / 6.2008-1243$

[18] Milos, F. S., and Rasky, D., "Review of Numerical Procedures for Computational Surface Thermochemistry," Journal of Thermophysics 
and Heat Transfer, Vol. 8, No. 1, 1994, pp. 24-34. doi: $10.2514 / 3.497$

[19] Martinelli, S., and Ruffin, S., "Validation Process for Blowing and Transpiration-Cooling in DPLR," AIAA Paper 2007-4255, 2007. doi: $10.2514 / 6.2007-4255$

[20] Emmons, H. W., and Leigh, D. C., "Tabulation of the Blasius Function with Blowing and Suction," Ministry of Supply, Aeronautical Research Council TR-CP-No.157, London, England, U.K., 1954.

[21] Kays, W. M., and Crawford, M.E., Convective Heat and Mass Transfer, 3rd ed., McGraw-Hill, New York, 1993.

[22] Zhang, H., Reggio, M., Trépanier, J.-Y., and Camarero, R., "Discrete Form of the GCL for Moving Meshes and Its Implementation in CFD Schemes," Computers and Fluids, Vol. 22, No. 1, 1993, pp. 9-23. doi:10.1016/0045-7930(93)90003-R

[23] Kim, K. H., Kim, C., and Rho, O.-H., "Methods for the Accurate Computations of Hypersonic Flows: II. Shock-Aligned Grid Technique," Journal of Computational Physics, Vol. 174, No. 1, 2001, pp. 81-119. doi:10.1006/jeph.2001.6896

[24] Saunders, D. A., Yoon, S., and Wright, M. J., "An Approach to Shock Envelope Grid Tailoring and Its Effect on Reentry Vehicle Solutions," AIAA Paper 2007-0207, 2007. doi: 10.2514/6.2007-0207

[25] Candler, G. V., Barnhardt, M., Drayna, T. W., Nompelis, I., Perterson, D. M., and Subbareddy, P., "Unstructured Grid Approaches for Accurate Aeroheating Simulations," AIAA Paper 2007-3959, 2007. doi: 10.2514/6.2007-3959

[26] Gnoffo, P. A., "Multi-Dimensional, Inviscid Flux Reconstruction for Simulation of Hypersonic Heating on Tetrahedral Grids," AIAA Paper 2009-0599, 2009. doi:10.2514/6.2009-599

[27] Kuntz, D., Hassan, B., and Potter, D., "Predictions of Ablating Hypersonic Vehicles Using an Iterative Coupled Fluid/Thermal Approach," Journal of
Thermophysics and Heat Transfer, Vol. 15, No. 2, 2001, pp. 129-139. doi: $10.2514 / 2.6594$

[28] Cohen, C. B., and Reshotko, E., "Similar Solutions for the Compressible Laminar Boundary Layer with Heat Transfer and Pressure Gradient," NACA TN-3325, 1955

[29] Alkandry, H., Boyd, I. D., and Martin, A., "Coupled Flow Field Simulations of Charring Ablators with Nonequilibrium Surface Chemistry," AIAA Paper 2013-2634, 2013. doi: $10.2514 / 6.2013-2634$

[30] Amar, A. J., "Modeling of One-Dimensional Ablation with Porous Flow Using Finite Control Volume Procedure," Master's Thesis, North Carolina State Univ., Raleigh, NC, 2006.

[31] Powars, C. A., and Kendall, R. M., Aerotherm Chemical Equilibrium (ACE) Computer Program - User's Manual, Aerotherm Corporation, Mountain View, CA, May 1969.

[32] Park, C., "On Convergence of Chemical Reacting Flows," AIAA Paper 1985-0427, 1985. doi:10.2514/6.1985-427

[33] Miller, M. A., Martin, A., and Bailey, S. C. C., "Investigation of the Scaling of Roughness and Blowing Effects on Turbulent Channel Flow," Experiments in Fluids, Vol. 55, No. 1675, 2014, pp. 1-11. doi:10.1007/s00348-014-1675-y

[34] Martin, A., and Boyd, I. D., "Assessment of Carbon-Phenolic-in-Air Chemistry Models for Atmospheric Re-Entry," AIAA Paper 2010$4656,2010$. doi: $10.2514 / 6.2010-5062$

G. Russell

Associate Editor 
This article has been cited by:

1. Alexandre Martin, Huaibao Zhang, Kaveh A. Tagavi. 2017. An introduction to the derivation of surface balance equations without the excruciating pain. International Journal of Heat and Mass Transfer 115, 992-999. [Crossref]

2. Alessandro Turchi, Pietro M. Congedo, Thierry E. Magin. 2017. Thermochemical ablation modeling forward uncertainty analysis?Part I: Numerical methods and effect of model parameters. International Journal of Thermal Sciences 118, 497-509. [Crossref]

3. Peter G. Cross, Iain D. Boyd. Conjugate Analysis of Rocket Nozzle Ablation . [Citation] [PDF] [PDF Plus]

4. Haoyue Weng, Alexandre Martin. Development of a Universal Solver and Its Application to Ablation Problems . [Citation] [PDF] [PDF Plus]

5. Joffrey Coheur, Alessandro Turchi, Pierre Schrooyen, Thierry Magin. Development of a unified model for flow-material interaction applied to porous charring ablators . [Citation] [PDF] [PDF Plus]

6. S. Peluchon, G. Gallice, L. Mieussens. 2017. A robust implicit-explicit acoustic-transport splitting scheme for two-phase flows. Journal of Computational Physics 339, 328-355. [Crossref]

7. Rui Fu, Haoyue Weng, Jonathan Wenk, Alexandre Martin. Application of A New Thermal-Mechanical Coupling Solver for Ablation . [Citation] [PDF] [PDF Plus]

8. Alexandre Martin, Iain D. Boyd. 2015. Modeling of Heat Transfer Attenuation by Ablative Gases During the Stardust Reentry. Journal of Thermophysics and Heat Transfer 29:3, 450-466. [Abstract] [Full Text] [PDF] [PDF Plus] 